\title{
Log(v) 3LPF: A Linear Power Flow Formulation for Unbalanced Three-Phase Distribution Systems
}

\author{
Ignacio Losada Carreño, Member, IEEE, Shammya Shananda Saha, Member, IEEE, Anna Scaglione, Fellow, IEEE \\ Daniel Arnold, Member, IEEE, Sy-Toan Ngo, Member, IEEE, Ciaran Roberts, Student Member, IEEE.
}

\begin{abstract}
In this work, we introduce $\log (\mathrm{v}) 3 \mathrm{LPF}$, a linear power flow solver for unbalanced three-phase distribution systems. $\log (v) 3 \mathrm{LPF}$ uses a logarithmic transform of the voltage phasor to linearize the $\mathrm{AC}$ power flow equations around the balanced case. We incorporate the modeling of ZIP loads, transformers, capacitor banks, switches and their corresponding controls and express the network equations in matrix-vector form. With scalability in mind, special attention is given to the computation of the inverse of the system admittance matrix, Ybus. We use the Sherman-Morrison-Woodbury identity for an efficient computation of the inverse of a rank-k corrected matrix and compare the performance of this method with traditional LU decomposition methods in terms of FLOPS. We showcase the solver for a variety of network sizes, ranging from tens to thousands of nodes, and compare the $\log (v)$ 3LPF with commercial-grade software, such as OpenDSS.
\end{abstract}

Index Terms-Distribution Systems Modeling, Linear Power Flow, Three-Phase Unbalanced, Transformer Modeling, Sherman-Morrison-Woodbury, Sparse Solvers

\section{INTRODUCTION}

A S smart grid technologies continue to proliferate, power distribution systems need to become better operated and controlled. The Power Flow (PF) problem plays an important role in the analysis and simulation of distribution power systems, e.g. in expansion planning as well as in optimizing the controls of the network, or in traditional state-estimation and contingency analysis applications. The study of the power flow problem dates back to the early 20th century when engineers used heuristics to operate the grid. A single-phase AC PF formulation was first seen in [1] and iterative methods for the nonlinear equations, such as Gauss-Siedel [1], Newton [2], Fast-Decoupled [3] or Backward-Forward Sweep [4], [5], were presented as a solution for modern computers to the power flow problem. Multi-phase modelling, introduced in [5] and also studied in [6], [7], is often required in distribution systems, as opposed to single-phase positive sequence, to capture the unbalanced characteristics of distribution networks. Relatively recently, exact convex relaxation techniques for radial systems have been developed to address the non-convex

Ignacio Losada Carreño, Shammya Shananda Saha, and Anna Scaglione are with the School of Electrical, Computer, and Energy Engineering, Arizona State University, Tempe, Arizona, USA. e-mail: ilosadac@asu.edu

Daniel Arnold,Sy-Toan Ngo, and Ciaran Roberts are with Lawrence Berkeley National Laboratory

This research was supported in part by the Director, Cybersecurity, Energy Security, and Emergency Response, Cybersecurity for Energy Delivery Systems program, of the U.S. Department of Energy, under contract DEAC02-05CH11231. Any opinions, findings, conclusions, or recommendations expressed in this material are those of the authors and do not necessarily reflect those of the sponsors of this work. characteristics of the AC Optimal Power Flow (OPF) problem, originally treated in [8]. These techniques may also work when solving three phase PF problems (c.f. [9]), however, the proposed methods do not necessarily lead themselves to efficient solvers.

Since the AC power flow problem was first formulated, multiple linearizations that remove the non-convexities and non-linearities have been proposed. The most widely used linearization relies on the DC assumptions [10], where voltages are nominal and the resistance of the line is neglected. The DC assumption has been proven to provide fast results at the cost of accuracy [11], specially in distribution systems where the voltage may deviate significantly from the nominal values. In the context of radial distribution systems, Baran and $\mathrm{Wu}$ [12] proposed a single-phase AC PF formulation, linear in the voltage and current squared. Other single-phase AC PF linearizations were proposed in [13]-[15]. The DistFlow equations were later expanded to three-phase unbalanced networks [16] while considering line losses. However, the linear DistFlow equations are limited to radial networks and the inclusion of losses in the formulation requires training of complex models. Also, the modeling of different network elements presents a challenge since the DistFlow equations are linear in the squared of the voltage and current magnitudes, and they do not consider voltage angles. New linearization techniques for multiphase network are shown in recent work [17]-[19]. For instance, the work from [17] assumes nominal voltages and no line losses. The authors in [18] use a single iteration of the fixed-point iteration method, and [19] uses curve fitting to approximate the nonlinear voltage ratios assuming small changes. The work from [17]-[19] did not consider the modeling of network elements such as multi-winding multiphase transformers and its multiple connections, or capacitor banks, switches, and the corresponding controls of all these devices. Additionally, some of these methods [17], [18] are tested on small networks and their ability to scale to realistic feeders is a question that remains unanswered. Furthermore, some linearizations rely on the radial assumption, and do not point to fast inversion methods when changes in the boundary conditions of the network tend to be sparse.

In this work, we provide an open-source three-phase unbalanced linear power flow solver, the $\log (v) 3 L P F$, that uses the $\mathrm{AC} \mathrm{PF}$ equations derived using first principles. We use inspiration from previous single-phase work in [20] to use a logarithmic transform of the voltage phasors. Expressing the the voltage phasor magnitude in logarithmic form enables a first-order Taylor expansion around zero of log-voltage magni- 
tude and log-voltage angle. We work under the assumption that a linear function centered around the balanced case will yield a good approximation. Moreover, the logarithmic transform technique has applications beyond radial networks, and could be generalized [20] for three-phase transmission networks. In addition to linearizing the power flow equations, we provide linear expressions to model ZIP loads, transformers and its multiple configurations, capacitors banks and switches, as well as control functionality for some of these devices. In general, changes in the controls are modeled as a perturbation of the system admittance matrix, $\mathbf{Y}_{\text {bus }}$. This new formulation is presented in a compact matrix-vector form, thus enabling further understanding of the impacts of changing boundary conditions to the network through a simple linear operator. We also provide attention to the efficiency of the solver, specially in the computation of the inverse of $\mathbf{Y}_{\text {bus }}$. Since changes in the operating condition of the network tend to be sparse, we leverage the Sherman-Morrison-Woodbury identity for an efficient computation of the inverse. We show results in terms of flops and provide a comparison with traditional LU decomposition methods that are used by commercial software [21]. Finally, we show results from running different IEEE radial networks, ranging from tens to thousands of nodes, and validate our solution against the OpenDSS software [22], that relies on iterative methods to provide an exact solution. A thorough validation of any linearization provides additional value, and is the basis to understand potential use cases. In fact, this work is motivated by the need of algorithms that can solve thousands of PF problems efficiently, to train reinforcement learning (RL) algorithms and learn control policies for the distribution system [23]. Potential applications are broad, ranging from cybersecurity [24] to applications on coupled transmission and distribution studies [25].

\section{Modeling OF MULTi-PHASE LINE ELEMENTS}

A distribution feeder can be modeled as an undirected graph $\mathcal{G}=\{\mathcal{N}, \mathcal{E}\}$ with $|\mathcal{N}|$ buses, and $|\mathcal{E}|$ number of edges. We denote $\Omega_{n}$ as the set of phases available in bus $n \in \mathcal{N}$, and $\Omega_{n m}$ is the set of phases on each edge $e=\{n, m\} \in \mathcal{E}$ connecting two buses $n, m \in \mathcal{N}$. It should be noted that $\left|\Omega_{n m}\right|=\left|\Omega_{m n}\right|$, and $\left|\Omega_{n m}\right| \leq \min \left(\left|\Omega_{n}\right|,\left|\Omega_{m}\right|\right) \leq 3$. We further divide the set of edges $\mathcal{E}$ into the set of distribution lines $\mathcal{E}_{l}$, the set of transformers $\mathcal{E}_{t}$, and the set of regulators $\mathcal{E}_{r}$ so that $\mathcal{E}=\mathcal{E}_{l} \cup \mathcal{E}_{t} \cup \mathcal{E}_{r}$. Each edge, $e \in \mathcal{E}$, is modeled as a series element using the $\Pi$-model representation to include the losses in a long distribution line as shown in Figure 1. In this section we use the term line or edge interchangeably to refer to any type edges, that is, distribution lines, transformers, or regulators. The equations presented here are derived from first principles, and are applicable to the three cases. We denote $v_{n}^{p}$ as the voltage phasor measured at bus $n \in \mathcal{N}$ and phase $p \in \Omega_{n}$, and $i_{n m, p}^{(n)}$ denotes the current phasor flowing from bus $n$ to bus $m, n, m \in \mathcal{N}$ on phase $p \in \Omega_{n m}$. $\mathbf{Y}_{n m} \in \mathbb{C}^{\left|\Omega_{n m}\right| \times\left|\Omega_{n m}\right|}$ denotes the admittance matrix of the line, and $\mathbf{Y}_{n m}^{s} \in \mathbb{C}^{\left|\Omega_{n m}\right| \times\left|\Omega_{n m}\right|}$ is the charging admittance, modeled as a shunt element, half of which is assumed to be located on each end of the line. The vector of current phasors

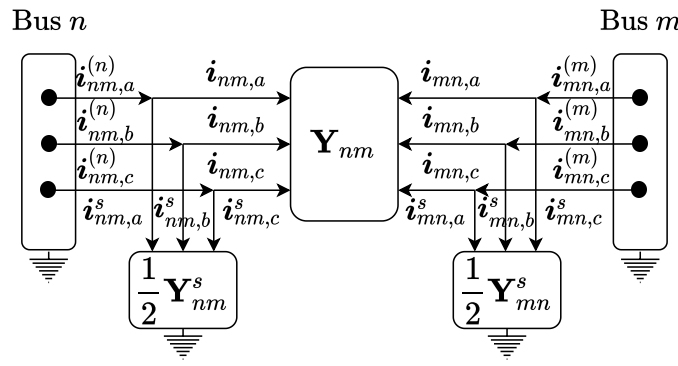

Fig. 1. П-model representation of a distribution line

in the line $\boldsymbol{i}_{n m} \in \mathbb{C}^{\left|\Omega_{n m}\right|}$ is related to the current flowing from bus $n \in \mathcal{N}$ to bus $m \in \mathcal{N}, \boldsymbol{i}_{n m}^{(n)} \in \mathbb{C}^{\left|\Omega_{n m}\right|}$, and shunt current $\boldsymbol{i}_{n m}^{s} \in \mathbb{C}^{\left|\Omega_{n m}\right|}$ by Kirchhoff's current law as follows

$$
\text { KCL: } \quad \boldsymbol{i}_{n m}^{(n)}=\boldsymbol{i}_{n m}+\boldsymbol{i}_{n m}^{s}
$$

where the dependency between $\boldsymbol{i}_{n m}, \boldsymbol{i}_{n m}^{s}$ and the vector of voltage phasors $\boldsymbol{v}_{n} \in \mathbb{C}^{\left|\Omega_{n}\right|}$ is given by Ohm's law as follows

$$
\begin{aligned}
\text { Ohm: } & \boldsymbol{i}_{n m}=\mathbf{Y}_{n m}^{(n)} \boldsymbol{v}_{n}+\mathbf{Y}_{n m}^{(m)} \boldsymbol{v}_{m} \\
\text { Losses: } & \boldsymbol{i}_{n m}^{s}=\frac{1}{2} \mathbf{Y}_{n m}^{s} \boldsymbol{v}_{n}
\end{aligned}
$$

Combining Eqs. (1)-(2), we obtain for every $e=\{n, m\} \in \mathcal{E}$ :

$$
\boldsymbol{i}_{n m}^{(n)}=\left(\mathbf{Y}_{n m}^{(n)}+\frac{1}{2} \mathbf{Y}_{n m}^{s}\right) \boldsymbol{v}_{n}+\mathbf{Y}_{n m}^{(m)} \boldsymbol{v}_{m}
$$

Note that, in general, for distribution lines $\mathbf{Y}_{n m}^{(n)}=-\mathbf{Y}_{n m}^{(m)}$ with the exception of transformers or regulators, as we will see later. The power flowing from bus $n \in \mathcal{N}$ to bus $m \in \mathcal{N}$ is $\boldsymbol{S}_{n m}^{(n)}=\boldsymbol{v}_{n}\left(\boldsymbol{i}_{n m}^{(n)}\right)^{H}$ and, in light of Eq. (3):

$$
\boldsymbol{S}_{n m}^{(n)}=\boldsymbol{v}_{n} \boldsymbol{v}_{n}^{H}\left(\mathbf{Y}_{n m}^{(n)}+\frac{1}{2} \mathbf{Y}_{n m}^{s}\right)^{H}+\boldsymbol{v}_{n} \boldsymbol{v}_{m}^{H}\left(\mathbf{Y}_{n m}^{(m)}\right)^{H}
$$

where the superscript $H$ stands for complex conjugate transpose, or Hermitian. Similarly, we can write the power flow flowing from $m \in \mathcal{N}$ to $n \in \mathcal{N}$ swapping the two indexes to obtain $\boldsymbol{S}_{m n}^{(m)}$. The non-linearity of the equations originates from the outer products $\boldsymbol{v}_{n} \boldsymbol{v}_{n}^{H}, \boldsymbol{v}_{n} \boldsymbol{v}_{m}^{H}$. In the following section, we present a method to approximate these outer products that leads to linear equations.

\section{A. Linearizing the three-phase unbalanced AC PF equations}

Every complex number $z$ can be expressed in polar form as $z=r e^{j \theta}$ where $r$ is the modulus and $\theta$ is the angle with respect to the real axis. Similarly, we can express the modulus of the complex number as $r=e^{\log (r)}$. We denote $u_{n}^{p}+j \theta_{n}^{p}:=$ $\log v_{n}^{p}$ as the logarithmic transformation of the voltage phasor $v_{n}^{p}$ on phase $p \in \Omega_{n}$ as shown in [20]. When $\left|\Omega_{n}\right|=3$, we can express $\boldsymbol{v}_{n}$ as:

$$
\boldsymbol{v}_{n}:=\left[e^{u_{n}^{a}} e^{j \theta_{n}^{a}}, e^{u_{n}^{b}} e^{j \theta_{n}^{b}}, e^{u_{n}^{c}} e^{j \theta_{n}^{c}}\right]^{T}
$$

In general, we know that phase differences in balanced distribution systems are $2 \pi / 3$ radians or 120 degrees, i.e. $\overline{\boldsymbol{\theta}}_{n}=[0,-2 \pi / 3,2 \pi / 3], n \in \mathcal{N}$ radians. In unbalanced 
systems, we know the voltage angle $\boldsymbol{\theta}_{n} \neq \overline{\boldsymbol{\theta}}_{n}$ will deviate from the balanced case. Let the operator $\operatorname{diag}($.$) return a$ square matrix with the elements of the vector in the diagonal or a block diagonal if the input is an array of matrices. Also, define $\boldsymbol{\Delta}_{n}^{(3)}:=\operatorname{diag}(\boldsymbol{\delta}), \boldsymbol{\delta}:=\left[1, e^{-j 2 \pi / 3}, e^{j 2 \pi / 3}\right]^{T}$ and $\tilde{\boldsymbol{\theta}}_{n}:=\left[\theta_{n}^{a}, \theta_{n}^{b}+\frac{2 \pi}{3}, \theta_{n}^{c}-\frac{2 \pi}{3}\right]$. By adding and subtracting from the phase angle in $\boldsymbol{v}_{n}$, we obtain:

$$
\boldsymbol{v}_{n}:=\left[\begin{array}{c}
e^{u_{n}^{a}} e^{j \theta_{n}^{a}} \\
e^{u_{n}^{b}} e^{j \theta_{n}^{b}+\frac{2 \pi}{3}-\frac{2 \pi}{3}} \\
e^{u_{n}^{c}} e^{j \theta_{n}^{c}-\frac{2 \pi}{3}+\frac{2 \pi}{3}}
\end{array}\right]=\boldsymbol{\Delta}_{n}^{(3)} \operatorname{diag}\left(\begin{array}{c}
e^{u_{n}^{a}} \\
e^{u_{n}^{b}} \\
e^{u_{n}^{c}}
\end{array}\right)\left[\begin{array}{c}
e^{j \tilde{\theta}_{n}^{a}} \\
e^{j \tilde{\theta}_{n}^{b}} \\
e^{j \tilde{\theta}_{n}^{c}}
\end{array}\right] .
$$

We assume that voltages are centered around 1 p.u. Thus, since we have defined $u_{n}^{p}:=\log \left|v_{n}^{p}\right|$ where $\left|v_{n}^{p}\right|$ is the modulus of the voltage phasor, and $\log (1)=0$, the first-order Taylor expansion of $\boldsymbol{v}_{n}$ is:

$$
\boldsymbol{v}_{n} \approx \boldsymbol{\Delta}_{n}^{(3)} \operatorname{diag}\left(\mathbb{1}+\boldsymbol{u}_{n}\right) e^{j \tilde{\boldsymbol{\theta}}_{n}}
$$

where $\mathbb{1}$ denotes the all-ones vector. If we substitute Eq. (7) in Eq. (4), the outer products $\boldsymbol{v}_{n} \boldsymbol{v}_{m}^{H}$ are:

$$
\boldsymbol{v}_{n} \boldsymbol{v}_{m}^{H} \approx \boldsymbol{\Delta}_{n}^{(3)} \operatorname{diag}\left(\mathbb{1}+\boldsymbol{u}_{n}\right) e^{j\left(\tilde{\boldsymbol{\theta}}_{n} \mathbb{1}^{T}-\mathbb{1} \tilde{\boldsymbol{\theta}}_{m}^{T}\right)} \operatorname{diag}\left(\mathbb{1}+\boldsymbol{u}_{m}\right)\left(\boldsymbol{\Delta}_{m}^{(3)}\right)^{H}
$$

Since we know that the matrices $\left(\tilde{\boldsymbol{\theta}}_{n} \mathbb{1}^{T}-\mathbb{1} \tilde{\boldsymbol{\theta}}_{m}^{T}\right)$ of phase differences are small, we can further expand $e^{j x} \approx 1+j x$ and dropping the second order terms, we obtain:

$$
\boldsymbol{v}_{n} \boldsymbol{v}_{m}^{H} \approx \boldsymbol{\Delta}_{n}^{(3)}\left(\mathbf{J}+\boldsymbol{u}_{n} \mathbb{1}^{T}+\mathbb{1} \boldsymbol{u}_{m}^{T}+j \tilde{\boldsymbol{\theta}}_{n} \mathbb{1}^{T}-j \mathbb{1} \tilde{\boldsymbol{\theta}}_{m}^{T}\right)\left(\boldsymbol{\Delta}_{m}^{(3)}\right)^{H}
$$

where $\mathbf{J}=\mathbb{1}^{T}$ is the all-ones matrix. Eq. (8) is linear in $\boldsymbol{u}$ and $\tilde{\boldsymbol{\theta}}$. We substitute Eq. (8) in Eq. (4), reorganize the terms and consider only the diagonal elements in $\mathbf{S}_{n m}^{(n)}$ and $\mathbf{S}_{m n}^{(m)}$, $\mathbf{s}_{n m} \triangleq\left[D\left(\mathbf{S}_{n m}^{(n)}\right), D\left(\mathbf{S}_{m n}^{(m)}\right)\right]^{T}$ where $D($.$) is the operator$ that returns the diagonal values of a matrix. If we denote the vector of state variables as $\boldsymbol{u}_{n m} \triangleq\left[\boldsymbol{u}_{n}, \boldsymbol{u}_{m}\right]^{T}$, and $\tilde{\boldsymbol{\theta}}_{n m} \triangleq$ $\left[\tilde{\boldsymbol{\theta}}_{n}, \tilde{\boldsymbol{\theta}}_{m}\right]$, we can express the linear power flow equation in matrix-vector form as follows:

$$
\mathbf{s}_{n m} \approx \mathbf{Y}_{n m}^{\boldsymbol{u}} \boldsymbol{u}_{n m}+\mathbf{Y}_{n m}^{\tilde{\boldsymbol{\theta}}} \tilde{\boldsymbol{\theta}}_{n m}+\tilde{\mathbf{y}}_{n m}
$$

where:

$\mathbf{Y}_{n m}^{\boldsymbol{u}}:=\tilde{\mathbf{Y}}_{n m}+\operatorname{diag}\left(\tilde{\mathbf{y}}_{n m}\right), \quad \mathbf{Y}_{n m}^{\tilde{\boldsymbol{\theta}}}:=-j \tilde{\mathbf{Y}}_{n m}+j \operatorname{diag}\left(\tilde{\mathbf{y}}_{n m}\right)$, $\tilde{\mathbf{y}}_{n m}:=\tilde{\mathbf{Y}}_{n m} \mathbb{1}$.

The matrix $\tilde{\mathbf{Y}}_{n m}$ is defined in the following expression:

$$
\tilde{\mathbf{Y}}_{n m}:=\boldsymbol{\Delta}_{n m}^{(3)}\left(\mathbf{Y}_{n m}^{\prime}\right)^{*}\left(\boldsymbol{\Delta}_{n m}^{(3)}\right)^{H}, \boldsymbol{\Delta}_{n m}^{(3)}:=\operatorname{diag}\left(\left[\boldsymbol{\Delta}_{n}^{(3)}, \boldsymbol{\Delta}_{m}^{(3)}\right]\right)
$$

where the symbol $*$ denotes the complex conjugate and the matrix $\mathbf{Y}_{n m}^{\prime}$ is known as the primitive admittance matrix, i.e.

$$
\mathbf{Y}_{n m}^{\prime}:=\left[\begin{array}{cc}
\mathbf{Y}_{n m}^{(n)}+\frac{1}{2} \mathbf{Y}_{n m}^{s} & \mathbf{Y}_{n m}^{(m)} \\
\mathbf{Y}_{m n}^{(n)} & \mathbf{Y}_{m n}^{(m)}+\frac{1}{2} \mathbf{Y}_{n m}^{s}
\end{array}\right]
$$

In Eq. (9), we have expressed the power flow equations in terms of the power flowing in the line and the nodal voltages. This is inconvenient, since we want to be able to relate the

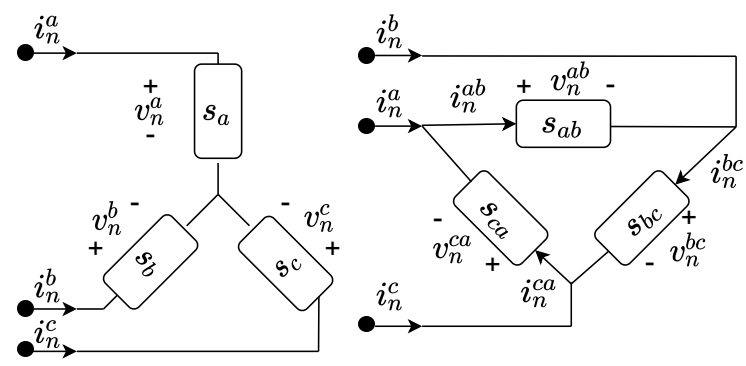

Fig. 2. Wye (left) and delta-connected (right) load

vector of power drawn by the loads to the nodal voltages. Stacking all the vectors $\boldsymbol{u}_{e}, \tilde{\boldsymbol{\theta}}_{e}, \boldsymbol{s}_{e}, \tilde{\boldsymbol{y}}_{e}, \forall e=\{n, m\} \in \mathcal{E}$ as:

$$
\underline{\boldsymbol{u}}:=\left[\begin{array}{c}
\boldsymbol{u}_{1} \\
\vdots \\
\boldsymbol{u}_{|\mathcal{E}|}
\end{array}\right], \underline{\tilde{\boldsymbol{\theta}}}:=\left[\begin{array}{c}
\tilde{\boldsymbol{\theta}}_{1} \\
\vdots \\
\tilde{\boldsymbol{\theta}}_{|\mathcal{E}|}
\end{array}\right], \underline{\boldsymbol{s}}:=\left[\begin{array}{c}
\boldsymbol{s}_{1} \\
\vdots \\
\boldsymbol{s}_{|\mathcal{E}|}
\end{array}\right], \underline{\tilde{y}}:=\left[\begin{array}{c}
\tilde{\boldsymbol{y}}_{1} \\
\vdots \\
\tilde{\boldsymbol{y}}_{|\mathcal{E}|}
\end{array}\right]
$$

and we place $\mathbf{Y}_{e}^{u}, \mathbf{Y}_{e}^{\tilde{\theta}}, e \in \mathcal{E}$ in a block diagonal matrix

$\mathbf{Y}^{\boldsymbol{u}}:=\operatorname{diag}\left(\left[\mathbf{Y}_{1}^{\boldsymbol{u}}, \ldots, \mathbf{Y}_{|\mathcal{E}|}^{\boldsymbol{u}}\right]\right), \mathbf{Y}^{\tilde{\boldsymbol{\theta}}}:=\operatorname{diag}\left(\left[\mathbf{Y}_{1}^{\tilde{\boldsymbol{\theta}}}, \ldots, \mathbf{Y}_{|\mathcal{E}|}^{\tilde{\boldsymbol{\theta}} \mid}\right]\right)$,

we can write:

$$
\underline{s} \approx \mathbf{Y}^{u} \underline{u}+\mathbf{Y}^{\tilde{\theta}} \underline{\tilde{\theta}}+\underline{\tilde{y}}
$$

thus, completing the derivation of our linear affine mapping. Before deriving a final expression that relates the vector of power injections and the nodal voltages through the system admittance matrix, i.e. $\mathbf{Y}_{\text {bus }}$, we introduce the models that account for the different characteristics of loads. These models, known as ZIP load models, consider the constant impedance (Z), constant current (I) and constant power (P) behavior of a load. We present these models in matrix-vector form to incorporate them into the calculation of the solution.

\section{Modeling Multi-Phase ZIP LoAdS}

In general, multi-phase loads can be modeled as phase-tophase in a delta connection or as phase-to-neutral in a wye connection as shown in Fig. 2. Let $\mathcal{L}$ denote the set of loads present in the network. We can define the set of wye-connected loads as $\mathcal{L}_{\mathrm{Y}}=\mathcal{L}_{\mathrm{Y}}^{\mathrm{Z}} \cup \mathcal{L}_{\mathrm{Y}}^{i} \cup \mathcal{L}_{\mathrm{Y}}^{p}$ where $\mathcal{L}_{\mathrm{Y}}^{\mathrm{Z}}, \mathcal{L}_{\mathrm{Y}}^{i}, \mathcal{L}_{\mathrm{Y}}^{p}$ are the set of constant impedance, constant current and constant power wyeconnected loads, respectively. Also, $\mathcal{L}_{\Delta}=\mathcal{L}_{\Delta}^{\mathrm{z}} \cup \mathcal{L}_{\Delta}^{i} \cup \mathcal{L}_{\Delta}^{p}$ is the set of delta-connected loads. Each load $\ell \in \mathcal{L}$ can be single phase, $\left|\Omega_{\ell}\right|=1$, two-phase $\left|\Omega_{\ell}\right|=2$ or three-phase $\left|\Omega_{\ell}\right|=3$, but it cannot have more phases than the bus it is connected to. We model loads $\ell \in \mathcal{L}$ as elements that draw power $\mathbf{s}_{\ell} \in \mathbb{C}^{\left|\Omega_{\ell}\right|}$ from the network given the voltage $\boldsymbol{v}_{\ell} \in \mathbb{C}^{\left|\Omega_{\ell}\right|}$. Loads can be constant impedance, constant current or constant power loads or a combination of the three. In this section, we introduce the models in matrix-vector form.

\section{A. Modeling Wye-connected loads}

Power can be drawn by a constant-impedance $s_{\ell}, \ell \in \mathcal{L}_{\mathrm{Y}}^{\mathrm{z}}$, constant current $s_{\ell}, \ell \in \mathcal{L}_{\mathrm{Y}}^{i}$, constant-power $s_{\ell}, \ell \in \mathcal{L}_{\mathrm{Y}}^{p}$ wyeconnected load. Next, we provide linear expressions for the power drawn by a wye-connected load. 
1) Constant Impedance (Z): Constant impedance loads draw power at different rates to maintain constant admittance of the load, $\mathbf{y}_{\ell}, \ell \in \mathcal{L}_{\mathrm{Y}}^{\mathrm{z}}$. As shown in Eq. (4) we can calculate the power drawn by a constant-impedance load $\ell \in \mathcal{L}_{\mathrm{Y}}^{\mathrm{Z}}$ as follows

$$
\begin{aligned}
\mathbf{s}_{\ell} & =\mathrm{D}\left(\boldsymbol{v}_{\ell} \boldsymbol{v}_{\ell}^{H} \operatorname{diag}\left(\mathbf{y}_{\ell}\right)^{*}\right) \approx \operatorname{diag}\left(\mathbb{1}+2 \boldsymbol{u}_{\ell}\right)\left(\mathbf{y}_{\ell}\right)^{*} \\
& \approx \mathbf{y}_{\ell}^{*}+2 \mathbf{Y}_{\ell}^{*} \boldsymbol{u}_{\ell}
\end{aligned}
$$

where $\mathbf{Y}_{\ell}:=\operatorname{diag}\left(\mathbf{y}_{\ell}\right), \mathbf{Y}_{\ell} \in \mathbb{C}^{\left|\Omega_{\ell}\right| \times\left|\Omega_{\ell}\right|}$.

2) Constant Current (I): Constant current loads adjust the voltage angle and keep the current of the load, $\boldsymbol{i}_{\ell}, \ell \in \mathcal{L}_{\mathrm{Y}}^{i}$ constant. We obtain an expression for constant-current loads $\ell \in \mathcal{L}_{\mathrm{Y}}^{i}$ as follows

$$
\begin{aligned}
\mathbf{s}_{\ell} & =\operatorname{diag}\left(\boldsymbol{i}_{\ell}\right)^{*} \boldsymbol{v}_{\ell} \approx \boldsymbol{\Delta}_{\ell}^{(3)} \operatorname{diag}\left(\boldsymbol{i}_{\ell}\right)^{*}\left(\mathbb{1}+\boldsymbol{u}_{\ell}+j \tilde{\boldsymbol{\theta}}_{\ell}\right) \\
& \approx \boldsymbol{\iota}_{\ell}+\mathbf{I}_{\ell} \boldsymbol{u}_{\ell}+j \mathbf{I}_{\ell} \tilde{\boldsymbol{\theta}}_{\ell}
\end{aligned}
$$

where $\boldsymbol{\iota}_{\ell}:=\boldsymbol{\Delta}_{\ell}^{(3)} \boldsymbol{i}_{\ell}^{*}, \mathbf{I}_{\ell}:=\operatorname{diag}\left(\boldsymbol{\iota}_{\ell}\right)$ and $\boldsymbol{\Delta}_{\ell}^{(3)}$ contains the entries of $\boldsymbol{\Delta}^{(3)}$ corresponding to the load phases.

3) Constant Power $(P)$ : The modeling of constant power loads is trivial since the power flow equations are expressed in terms of power injections. Thus, we keep the power drawn by the load, $s_{\ell}, \ell \in \mathcal{L}_{\mathrm{Y}}^{p}$ constant. In the following subsection, we derive analogous expressions for delta-connected loads.

\section{B. Modeling Delta-connected loads}

Power is drawn by a constant-impedance $s_{\ell}, \ell \in \mathcal{L}_{\Delta}^{\mathrm{z}}$, constant-current $s_{\ell}, \ell \in \mathcal{L}_{\Delta}^{i}$, constant-power $s_{\ell}, \ell \in \mathcal{L}_{\Delta}^{p}$ delta-connected load. Delta-connected loads differ from wyeconnected loads in that phase-to-phase readings need to be converted to line-to-neutral. Loads draw line current $\boldsymbol{i}_{\ell}, \ell \in$ $\Omega_{\ell}$ at a certain line-to-neutral voltage $\boldsymbol{v}_{\ell}$. We may express the vector of line currents drawn by a delta-connected load $\boldsymbol{i}_{\ell}, \ell \in \mathcal{L}_{\Delta}$ as a function of the vector of phase-to-phase currents, $\boldsymbol{i}_{\ell}^{\Delta}, \ell \in \mathcal{L}_{\Delta}$. When $\left|\Omega_{\ell}\right|=3, \boldsymbol{i}_{\ell}^{\Delta}=\left[i_{\ell}^{a b}, i_{\ell}^{b c}, i_{\ell}^{c a}\right]$ and we can use Kirchhoff's law to obtain the linear mapping between $\boldsymbol{i}_{\ell}$ and $\boldsymbol{i}_{\ell}^{\Delta}$ as follows

$$
\boldsymbol{i}_{\ell}=\boldsymbol{\Pi}_{\ell} \boldsymbol{i}_{\ell}^{\Delta}, \quad \text { where } \quad \boldsymbol{\Pi}_{\ell}=\left(\begin{array}{ccc}
1 & 0 & -1 \\
-1 & 1 & 0 \\
0 & -1 & 1
\end{array}\right)
$$

Similarly, we can find the mapping between line-to-neutral $\boldsymbol{v}_{\ell}$ and line-to-line voltages $\boldsymbol{v}_{\ell}^{\Delta}$. When $\left|\Omega_{\ell}\right|=3, \boldsymbol{v}_{\ell}^{\Delta}=$ $\left[v_{\ell}^{a b}, v_{\ell}^{b c}, v_{\ell}^{c a}\right]$ and we can use the expression $\boldsymbol{v}_{\ell}^{\Delta}=\boldsymbol{\Pi}^{T} \boldsymbol{v}_{\ell}$. Finally, we use the transformation matrix $\boldsymbol{\Lambda}$ from [26] to map the power drawn by a delta-connected load $s_{\ell}^{\Delta}, \ell \in \mathcal{L}_{\Delta}$ to the power drawn from the line $s_{\ell}$. When $\left|\Omega_{\ell}\right|=3$,

$\boldsymbol{s}_{\ell}=\boldsymbol{\Lambda}_{\ell} \boldsymbol{s}_{\ell}^{\Delta}$, and $\boldsymbol{\Lambda}_{\ell}=\frac{\sqrt{3}}{3}\left(\begin{array}{ccc}e^{-j \pi / 6} & 0 & e^{j \pi / 6} \\ e^{j \pi / 6} & e^{-j \pi / 6} & 0 \\ 0 & e^{j \pi / 6} & e^{-j \pi / 6}\end{array}\right)$

Expressions for delta-connected ZIP loads are presented next
1) Constant Impedance (Z): The power drawn by a constant-impedance load $\mathbf{s}_{\ell}, \ell \in \mathcal{L}_{\Delta}^{\mathrm{z}}$ is given as

$$
\begin{aligned}
\boldsymbol{i}_{\ell}^{\Delta} & =\operatorname{diag}\left(\mathbf{y}_{\ell}^{\Delta}\right) \boldsymbol{v}_{\ell}^{\Delta} \\
\boldsymbol{i}_{\ell} & =\boldsymbol{\Pi}_{\ell} \operatorname{diag}\left(\mathbf{y}_{\ell}^{\Delta}\right) \boldsymbol{\Pi}_{\ell}^{T} \boldsymbol{v}_{\ell} \\
\mathbf{s}_{\ell} & =\mathrm{D}\left(\boldsymbol{v}_{\ell} \boldsymbol{i}_{\ell}^{H}\right)=\mathrm{D}\left(\boldsymbol{v}_{\ell} \boldsymbol{v}_{\ell}^{H} \boldsymbol{\Pi}_{\ell} \operatorname{diag}\left(\mathbf{y}_{\ell}^{\Delta}\right)^{*} \boldsymbol{\Pi}_{\ell}^{T}\right)
\end{aligned}
$$

Furthermore, we define the constants:

$$
\mathbf{Y}_{\ell}:=\left(\boldsymbol{\Delta}_{\ell}^{(3)}\right)^{*} \boldsymbol{\Pi}_{\ell} \operatorname{diag}\left(\mathbf{y}_{\ell}^{\Delta}\right) \boldsymbol{\Pi}_{\ell}^{T} \boldsymbol{\Delta}_{\ell}^{(3)}, \quad \mathbf{y}_{\ell}=\mathbf{Y}_{\ell} \mathbb{1}
$$

Substituting Eq. (8) in Eq. (20), taking only the diagonal elements, we obtain

$$
\mathbf{s}_{\ell} \approx \mathbf{y}_{\ell}^{*}+\left(\mathbf{Y}_{\ell}^{*}+\operatorname{diag}\left(\mathbf{y}_{\ell}^{*}\right)\right) \boldsymbol{u}_{\ell}+j\left(\mathbf{Y}_{\ell}^{*}-\operatorname{diag}\left(\mathbf{y}_{\ell}^{*}\right)\right) \tilde{\boldsymbol{\theta}}_{\ell}
$$

2) Constant Current (I): The power drawn by a constantcurrent load $\mathbf{s}_{\ell}, \ell \in \mathcal{L}_{\Delta}^{i}$ can be obtained as follows

\begin{tabular}{|c|c|c|c|}
\hline ZIP & Phases & Wye-connected & Delta-connected \\
\hline $\mathbf{Z}$ & $\begin{array}{c}\left|\Omega_{\ell}\right|=1,3 \\
\left|\Omega_{\ell}\right|=2\end{array}$ & $\begin{array}{c}\mathbf{y}_{\ell}=\frac{\left|\bar{v}_{\ell}\right|^{2}}{\left(\bar{s}_{\ell}\right)^{*}} \mathbb{1}_{\left|\Omega_{\ell}\right|} \\
\mathbf{y}_{\ell}=\frac{2}{3} \frac{\left|\bar{v}_{\ell}\right|^{2}}{\left(\bar{s}_{\ell}\right)^{*}} \mathbb{1}_{\left|\Omega_{\ell}\right|}\end{array}$ & $\begin{array}{c}\overline{\mathbf{y}_{\ell}^{\Delta}}=\frac{\left|\bar{v}_{\ell}\right|^{2}}{\left(\bar{s}_{\ell}\right)^{*}} \mathbb{1}_{\left|\Omega_{\ell}\right|} \\
\mathbf{y}_{\ell}^{\Delta}=\frac{2}{3} \frac{\left|\bar{v}_{\ell}\right|^{2}}{\left(\bar{s}_{\ell}\right)^{*}} \mathbb{1}_{\left|\Omega_{\ell}\right|}\end{array}$ \\
\hline $\mathbf{I}$ & $\begin{array}{c}\left|\Omega_{\ell}\right|=1 \\
\left|\Omega_{\ell}\right|=2,3\end{array}$ & $\begin{array}{c}\boldsymbol{i}_{\ell}=\frac{1}{\left|\Omega_{\ell}\right|} \frac{\left|\bar{s}_{\ell}\right|}{\left|\bar{v}_{\ell}\right|} \mathbb{1}_{\left|\Omega_{\ell}\right|} \\
\boldsymbol{i}_{\ell}=\frac{1}{\sqrt{3}\left|\Omega_{\ell}\right|} \frac{\left|\bar{s}_{\ell}\right|}{\left|\bar{v}_{\ell}\right|} \mathbb{1}_{\left|\Omega_{\ell}\right|}\end{array}$ & $\begin{aligned} i_{\ell}^{\Delta} & =\frac{1}{\left|\Omega_{\ell}\right|} \frac{\left|\bar{s}_{\ell}\right|}{\left|\bar{v}_{\ell}\right|} \mathbb{1}_{\left|\Omega_{\ell}\right|} \\
\boldsymbol{i}_{\ell}^{\Delta} & =\frac{1}{\left|\Omega_{\ell}\right|} \frac{\left|\bar{s}_{\ell}\right|}{\left|\bar{v}_{\ell}\right|} \mathbb{1}_{\left|\Omega_{\ell}\right|}\end{aligned}$ \\
\hline $\mathbf{P}$ & $\left|\Omega_{\ell}\right|=1,2,3$ & $s_{\ell}=\frac{s_{\ell}}{\left|\Omega_{\ell}\right|} \mathbb{1}_{\left|\Omega_{\ell}\right|}$ & $\boldsymbol{s}_{\ell}^{\Delta}=\frac{s_{\ell}}{\left|\Omega_{\ell}\right|} \mathbb{1}_{\left|\Omega_{\ell}\right|}$ \\
\hline
\end{tabular}

$$
\begin{aligned}
\mathbf{s}_{\ell} & =\operatorname{diag}\left(\boldsymbol{\Pi}_{\ell} \boldsymbol{i}_{\ell}^{\Delta}\right)^{*} \boldsymbol{v}_{\ell} \\
& \approx \boldsymbol{\Delta}_{\ell}^{(3)} \operatorname{diag}\left(\boldsymbol{\Pi}_{\ell} \boldsymbol{i}_{\ell}^{\Delta}\right)^{*}\left(\mathbf{1}+\boldsymbol{u}_{\ell}+j \tilde{\boldsymbol{\theta}}_{\ell}\right) \approx \boldsymbol{\iota}_{\ell}+\mathbf{I}_{\ell} \boldsymbol{u}_{\ell}+j \mathbf{I}_{\ell} \tilde{\boldsymbol{\theta}}_{\ell}
\end{aligned}
$$

where $\boldsymbol{\iota}_{\ell}:=\boldsymbol{\Delta}_{\ell}^{(3)} \boldsymbol{\Pi}_{\ell}\left(\boldsymbol{i}_{\ell}^{\Delta}\right)^{*}$ and $\mathbf{I}_{\ell}=\operatorname{diag}\left(\boldsymbol{\iota}_{\ell}\right)$.

3) Constant Power $(P)$ : The expression for the power drawn from the line can be obtained by using the transformation matrix, i.e. $\mathbf{s}_{\ell} \approx \boldsymbol{\Lambda}_{\ell} \mathbf{s}_{\ell}^{\Delta}, \ell \in \mathcal{L}_{\Delta}^{p}$, completing the derivation of the linear expressions for ZIP loads.

TABLE I

ZIP LOAD CONSTANTS

\section{Relating the ZIP constants to the IEEE test cases}

All the linear expressions from Eqs. (16)-(23) use the ZIP constants, i.e. the wye-connected constants $\mathbf{y}_{\ell}, \boldsymbol{i}_{\ell}, \boldsymbol{s}_{\ell}, \ell \in \mathcal{L}_{\mathrm{Y}}$ and delta-connected constants $\mathbf{y}_{\ell}^{\Delta}, i_{\ell}^{\Delta}, s_{\ell}^{\Delta}, \ell \in \mathcal{L}_{\Delta}$, to model the different characteristics of the load. In general, a load $\ell \in \mathcal{L}$ is defined by its rated power $\bar{s}_{\ell}=\bar{p}_{\ell}+j \bar{q}_{\ell}$ in VAs and rated voltage readings $\left|\bar{v}_{\ell}\right|$ in Volts, as provided in all the IEEE test cases available in OpenDSS [27]. It should be noted that when $\left|\Omega_{\ell}\right|=1, \ell \in \mathcal{L}_{\mathrm{Y}}$, OpenDSS reports a lineto-neutral voltage. Every other time, the voltage is phase-tophase. Thus, when we refer to $\left|\bar{v}_{\ell}\right|$, we are referring to the value provided by OpenDSS in the definition of the load. Also, unbalanced loads must be defined as separate loads, since the voltage and power ratings will differ. Using these readings, we summarize the calculations of the ZIP constants in Table 


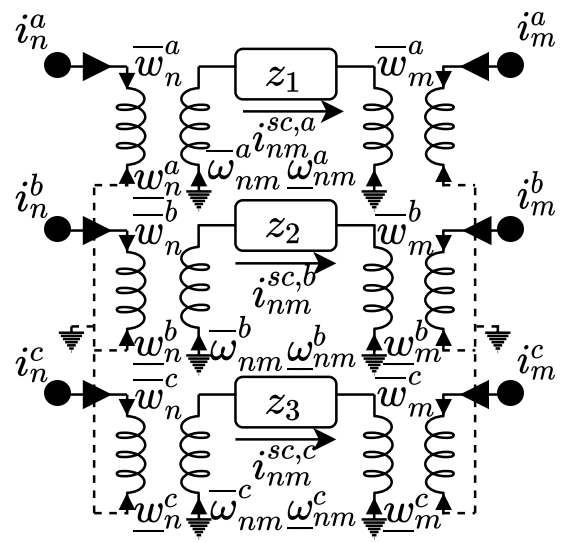

Fig. 3. 3-phase Wye-grounded Wye-grounded transformer model

I where $\mathbb{1}_{\left|\Omega_{\ell}\right|}$ is the vector of ones with $\left|\Omega_{\ell}\right|$ entries. In the following section, we model different power delivery elements and provide algorithms to control some of these elements.

\section{MODELING AND CONTROL OF POWER DELIVERY ELEMENTS}

In this section, we present the modeling of some standard equipment found in distribution systems. Modeling of high-voltage substation transformers, low voltage residential split-phase transformers, and voltage regulators as autotransformers with control functionality are presented. Capacitor banks with switching capabilities are represented as negative loads that can inject reactive power. Solar inverters are modeled as negative loads that can control the amount of apparent power injection. We first introduce the modeling of the different components and then incorporate the controls into the models.

\section{A. Modeling of power delivery elements}

1) Capacitor Banks: Capacitor banks are modelled as wyeconnected or delta-connected constant impedance loads that inject reactive power to the network. Let $\mathcal{C}$ denote the set of the capacitors on the network. We further divide $\mathcal{C}=\mathcal{C}_{\mathrm{Y}} \cup$ $\mathcal{C}_{\Delta}$ into wye-connected $\mathcal{C}_{\mathrm{Y}}$ and delta-connected $\mathcal{C}_{\Delta}$ capacitors. The impedance matrix of any capacitor is given by $y_{c}, c \in \mathcal{C}_{\mathrm{Y}}$ in a wye connection or $y_{c}, c \in \mathcal{C}_{\Delta}$ in a delta connection. The calculation follows from Eqs. (16), (22) and Table I. The rated voltage of the capacitor $\left|v_{c}\right|$ is analogous to $\left|v_{\ell}\right|$ in a load, and the rated power $s_{c}=-j q_{c}$ is analogous to $s_{\ell}$, where $q_{c}$ is the reactive power of the capacitor bank.

2) Three-phase transformers: We model multi-phase multiwinding transformers as line elements with line admittances, such as those shown in Eq. (4), which enables the use of Eqs. (1)-(12) to model transformers. In the $\log (v) 3 L P F$ linearization, transformers are represented in the network through the primitive admittance matrix $\mathbf{Y}_{n m}^{\prime}$ as shown in Eq. (12). However, unlike in the case of a distribution line, the primitive admittance matrix of the transformer $\mathbf{Y}_{n m}^{\prime},\{n, m\} \in$ $\mathcal{E}_{t},\{n, m\} \notin \mathcal{E}_{l}$ may not be symmetric. We follow the modeling from [28] to obtain the primitive matrix of the transformer. An example of a Wye-grounded Wye-grounded is shown in Fig. 3. In general, each transformer has a core, and its configuration is characterized by the connection of the transformer to both buses $n, m \in \mathcal{N}$. If the transformer has more than two windings, e.g. a split-phase 120/240 V transformer, one may apply superposition and treat each winding separately. Let $\boldsymbol{i}_{n m}^{s c} \in \mathbb{C}^{\left|\Omega_{n m}\right|}$ denote the short-circuit current measured on the transformer. The voltage drop $\boldsymbol{v}_{n m}^{s c}$ is related to $\boldsymbol{i}_{n m}^{s c}$ as follows

$$
\boldsymbol{i}_{n m}^{s c}=\mathbf{Y}_{n m} \boldsymbol{v}_{n m}^{s c}, \quad\{n, m\} \in \mathcal{E}_{t}
$$

where $\mathbf{Y}_{n m}=\mathbf{Z}_{n m}^{-1}=\operatorname{diag}\left(\left[1 / z_{1}, \ldots, 1 / z_{\left|\Omega_{n m}\right|}\right]^{T}\right)$, and $z_{i}, i \in \Omega_{n m},\{n, m\} \in \mathcal{E}_{t}$ is the short-circuit impedance in per-unit. Now, let $\boldsymbol{\omega}_{n m} \in \mathbb{C}^{2\left|\Omega_{n m}\right|}$ denote the vector of currents in the core of the transformer $\{n, m\} \in \mathcal{E}_{t}$. When $\left|\Omega_{n m}\right|=1, \omega_{n m}=\left[\bar{\omega}_{n m}^{p}, \underline{\omega}_{n m}^{p}\right]^{T}, p \in \Omega_{n m}$. The vector $\boldsymbol{\omega}_{n m}$ is related to the short-circuit current as $\boldsymbol{\omega}_{n m}=$ $\boldsymbol{\beta} \boldsymbol{i}_{n m}^{s c}$ where $\boldsymbol{\beta} \in\{-1,0,1\}^{2\left|\Omega_{n m}\right| \times\left|\Omega_{n m}\right|}$ is the incidence matrix that relates the two variables. We can also define $\boldsymbol{w}_{n m}=\left[\boldsymbol{w}_{n}, \boldsymbol{w}_{m}\right] \in \mathbb{C}^{4\left|\Omega_{n m}\right|}$ as the vector of currents in the winding. When $\left|\Omega_{n m}\right|=1, \boldsymbol{w}_{n}=\left[\bar{w}_{n}, \underline{w}_{n}\right]$ and $\boldsymbol{w}_{m}=\left[\bar{w}_{m}, \underline{w}_{m}\right]$. Also, $\boldsymbol{w}_{n m}=\mathbf{N} \boldsymbol{\omega}_{n m}$, where $\mathbf{N} \in$ $\{0,1,-1, \sqrt{3},-\sqrt{3}\}^{4\left|\Omega_{n m}\right| \times 2\left|\Omega_{n m}\right|}$ and the scaling factor $\sqrt{3}$ is used to go from line-to-line to line-to-neutral values. The vector of currents on both terminals $\boldsymbol{i}_{n m}=\left[\boldsymbol{i}_{n}, \boldsymbol{i}_{m}\right]^{T}$ can be expressed as $\boldsymbol{i}_{n m}=\mathbf{X} \boldsymbol{\iota}_{n m}$ where $\mathbf{X} \in \mathbb{C}^{2\left|\Omega_{n m}\right| \times 4\left|\Omega_{n m}\right|}$ is the matrix that maps winding current to terminal currents, and depends on the connection. It should be noted that Fig. 3 shows a Wye-grounded Wye-grounded transformer, but any configuration can be implemented through the matrix $\mathbf{X}$. Finally, we multiply Eq. (24) by the incidence matrices from the left, and multiplying by their transposes from the right, we can find the relationship between the terminal voltages $\boldsymbol{v}_{n m}=\left[\boldsymbol{v}_{n}, \boldsymbol{v}_{m}\right]^{T}$ and the terminal currents as follows

$$
\boldsymbol{i}_{n m}=\mathbf{X} \mathbf{N} \boldsymbol{\beta} \mathbf{Y}_{n m} \boldsymbol{\beta}^{\mathrm{T}} \mathbf{N}^{\mathrm{T}} \mathbf{X}^{\mathrm{T}} \boldsymbol{v}_{n m}, \quad\{n, m\} \in \mathcal{E}_{t}
$$

thus, $\mathbf{Y}_{n m}^{\prime}=\mathbf{X} \mathbf{N} \boldsymbol{\beta} \mathbf{Y}_{n m} \boldsymbol{\beta}^{\mathrm{T}} \mathbf{N}^{\mathrm{T}} \mathbf{X}^{\mathrm{T}}, \mathbf{Y}_{n m}^{\prime} \in \mathbb{C}^{2\left|\Omega_{n m}\right| \times 2\left|\Omega_{n m}\right|}$ is the primitive admittance matrix of the transformer, used in Eq. (11). Refer to [28] for an example on how to calculate the incidence matrices.

3) Distributed Energy Resources (DER): We model DER, in particular solar resources and batteries, as constant power wye-connected loads as in Eq. (16). The power injected to the system is time dependent, and is subject to fluctuations due to the available power produced in the case of solar panels. The power injected by solar plants and batteries can be controlled by the inverter, as we will see in the next section.

\section{B. Controlling power delivery elements}

1) Voltage regulators: We model a regulator $r=\{n, m\} \in$ $\mathcal{E}_{r}$ as a line element that connects two buses $n, m \in \mathcal{N}$. Voltage regulators are modeled as single-phase auto-transformers, $\left|\Omega_{r}\right|=1, r \in \mathcal{E}_{r}$. To represent the regulator controls, we use the modeling from [7], and include the controls $\boldsymbol{\Gamma}_{r}$ in the calculation of the primitive admittance matrix $\mathbf{Y}_{n m}^{\prime}$ as follows

$$
\mathbf{Y}_{n m}^{\prime}=\boldsymbol{\Gamma}_{r} \mathbf{X}_{r} \mathbf{N}_{r} \boldsymbol{\beta} \mathbf{Y}_{n m} \boldsymbol{\beta}^{\mathrm{T}} \mathbf{N}_{r}^{\mathrm{T}} \mathbf{X}_{r}^{\mathrm{T}} \boldsymbol{\Gamma}_{r}^{\mathrm{T}}, r=\{n, m\} \in \mathcal{E}_{r}
$$


where $\boldsymbol{\Gamma}_{r}=\operatorname{diag}^{-1}(\gamma)$ and $\gamma_{r}=\left[\gamma_{1}, \ldots, \gamma_{2\left|\Omega_{n m}\right|}\right] \in$ $[-0.9,1.1]^{2\left|\Omega_{n m}\right|}$ and $\gamma_{i}, i \in\left\{1, \ldots, 2\left|\Omega_{n m}\right|\right\}$ is defined as follows

$$
\gamma_{i}=1 \pm 0.00625 \tau_{i}
$$

where $\tau_{i} \in[-16,16] \cap \mathbb{Z}$ is the tap number. The coefficients in Eq. (27) are due to the fact that most regulators allow voltage to be controlled within $\pm 10 \%$ of its nominal value, in 32 steps. In general, the regulator controls the secondary voltage while tapping the voltage in the primary. Thus, $\gamma_{i}=$ $1, i \in\left\{1, \ldots,\left|\Omega_{n m}\right|\right\}$. To obtain the tap numbers, the regulator measures the voltage in the relay $\boldsymbol{v}_{r}$, given (28).

$$
\boldsymbol{v}_{r}=\frac{1}{\kappa} \boldsymbol{v}_{n}-\frac{z_{v}^{\prime}}{\xi_{p}} \boldsymbol{i}_{n}
$$

where $\kappa$ is the ratio between the winding voltage and the regulator control voltage, $\xi_{p}$ is the rated primary current, in Amps, and $z_{v}^{\prime}=r_{v}^{\prime}+j x_{v}^{\prime}$ is the line drop compensator setting, in Volts. The tap number is given as follows

$$
\boldsymbol{\tau}=\frac{1}{0.75} \min \left(\left|\boldsymbol{v}_{\text {reg }}-\frac{1}{2} \boldsymbol{v}_{b}-\boldsymbol{\nu}_{r}\right|,\left|\boldsymbol{v}_{\text {reg }}+\frac{1}{2} \boldsymbol{v}_{b}-\boldsymbol{\nu}_{r}\right|\right)
$$

where $\boldsymbol{v}_{\text {reg }}$ is the regulator setpoint in Volts, $\boldsymbol{v}_{b}$ is the bandwidth, and $\boldsymbol{\nu}_{r}$ is the voltage magnitude, $\boldsymbol{v}_{r}=\operatorname{diag}\left(\boldsymbol{\nu}_{r}\right) e^{j \boldsymbol{\theta}_{r}}$, where the values are given in Volts. In general, the voltage regulator setting is $120 \mathrm{~V}$, thus, each step in the tap amounts to $0.75 \mathrm{~V}$.

2) Capacitor controls: Let $s_{c}$ denote the number of steps in a shunt capacitor, $c \in \mathcal{C}$ with switches $\eta_{c, i} \in\{0,1\}$ that are to be controlled by our algorithm. The impedance values $y_{c}, y_{c}^{\Delta}$ to be used in the modeling of a constant impedance load are given as

$$
y_{c}=\sum_{i=1}^{s_{c}} \eta_{c, i} y_{c, i}^{\mathrm{Y}}, c \in \mathcal{C}_{\mathrm{Y}} \quad y_{c}^{\Delta}=\sum_{i=1}^{s_{c}} \eta_{c, i} y_{c, i}^{\Delta}, c \in \mathcal{C}_{\Delta}
$$

We may consider different types of controls, such as current, voltage, kvar, power factor, or time. The control value in the capacitor $\eta_{c, i}$ is given as

$$
\eta_{c, i}=u\left(\vartheta-\bar{\vartheta}_{c}\right)+u\left(\underline{\vartheta}_{c}-\vartheta\right)
$$

where $\vartheta$ is the control input, $\bar{\vartheta}_{c}, \underline{\vartheta}_{c}$ are the maximum and minimum limits, respectively, of the interval outside of which the control operates, and $u($.$) is the step function. If a voltage$ override mode is used, then $\eta_{c, i}$ is given as

$$
\eta_{c, i}=\left(u\left(\boldsymbol{v}-\underline{v}_{c}\right)-u\left(\boldsymbol{v}-\bar{v}_{c}\right)\right)\left(u\left(\vartheta-\bar{\vartheta}_{c}\right)+u\left(\underline{\vartheta}_{c}-\vartheta\right)\right)
$$

where $v$ is the voltage measured at some point in the network, and $\bar{v}_{c}, \underline{v}_{c}$ are the maximum and minimum voltage settings that allow for the operation of the control. Finally, the different types of controls that use $\vartheta$ in Eq. (31) may be obtained as follows

Current: $\vartheta=\frac{1}{\xi_{r}} \boldsymbol{i}_{n m}, \quad$ Voltage: $\vartheta=\frac{1}{\rho_{r}} \boldsymbol{v}_{n}$

kvar: $\vartheta=\operatorname{diag}\left(\boldsymbol{v}_{n}\right)\left(\boldsymbol{i}_{n m}^{(n)}\right)^{*}, \quad$ Time: $\vartheta=t$

Power Factor: $\vartheta=\operatorname{diag}^{-1}\left(\mathfrak{I}\left(\boldsymbol{s}_{n m}^{n}\right)\right) \mathfrak{R}\left(\boldsymbol{s}_{n m}^{n}\right)$ where $\xi_{r}, \rho_{r}$ are the ratio between the monitored line current and voltage and the corresponding current and voltage control, respectively. In general, the capacitor control measures the voltage $\boldsymbol{v}_{n}$ where the capacitor is connected $n \in \mathcal{N}$, or current and power values measured dowstream, $\boldsymbol{i}_{n m}^{(n)}, \boldsymbol{s}_{n m}^{n},\{n, m\} \in \mathcal{E}$ . Also, the operators $\mathfrak{R}(),. \mathfrak{I}($.$) return the real and imaginary$ part of the phasor, and $t$ denotes time.

3) Control of smart inverters: We model the control functionality of smart inverters using Volt-Var (VV) and VoltWatt (VW) schemes as shown in [23]. The power injected to the system is a function of a piece-wise function, or "droop" curves. This control scheme injects reactive power when voltage decreases and transitions to VAR consumption when the voltage is high. The VV-VW curves are given as follows

$$
\begin{gathered}
u_{i}^{p}=f_{i}^{p}(\tilde{v}) \triangleq \begin{cases}\tilde{p} & \bar{v} \in\left[0, \eta_{4}\right] \\
\left(\frac{\eta_{5}-\tilde{v}}{\eta_{5}-\eta_{4}}\right) \tilde{p} & \tilde{v} \in\left(\eta_{4}, \eta_{5}\right] \\
0 & \tilde{v} \in\left(\eta_{5}, \infty\right)\end{cases} \\
u_{i}^{q}=f_{i}^{q}(\tilde{v}) \triangleq \begin{cases}\tilde{q} & \tilde{v} \in\left[0, \eta_{1}\right] \\
\left(\frac{\eta_{2}-\tilde{v}}{\eta_{2}-\eta_{1}}\right) \tilde{q} & \tilde{v} \in\left(\eta_{1}, \eta_{2}\right] \\
0 & \tilde{v} \in\left(\eta_{2}, \eta_{3}\right) \\
-\left(\frac{\eta_{3}-\tilde{v}}{\eta_{4}-\eta_{3}}\right) \tilde{q} & \tilde{v} \in\left[\eta_{3}, \eta_{4}\right] \\
-\tilde{q} & \tilde{v} \in\left(\eta_{4}, \infty\right)\end{cases}
\end{gathered}
$$

where $\boldsymbol{\eta}=\left[\eta_{1}, \ldots, \eta_{5}\right]$ are the control settings or parameters that characterize the VV-VW curves, $\tilde{p}$ is the power that can be injected into the system. When modeling solar resources, $\tilde{p}$ in Eq. (34) is in general the maximum power produced by the panel $\tilde{p}=\bar{p}$ unless curtailment is necessary for the safe operation of the system. The calculation of $\bar{p}$ is out of the scope of this paper, but can be obtained using methods like in [29]. Also, $\tilde{q}$ is the available reactive power, and $\tilde{v}$ is a lowpass filtered version of the voltage magnitude, introduced to avoid undesired controls caused by noisy measurements. The quantities $\tilde{q}$ and $\tilde{v}$ are given as follows.

$$
\begin{aligned}
\tilde{v}_{i, t} & =\tilde{v}_{i, t-1}+\tau_{i}^{m}\left(v_{i, t}-\tilde{v}_{i, t-1}\right) \\
\tilde{q} & \leq \sqrt{\bar{s}^{2}-f^{p}(\tilde{v})^{2}}
\end{aligned}
$$

where $\tau_{i}^{m}$ is a time constant, $\bar{s}$ is the capacity of the inverter, and $v_{i, t}$ is the measured voltage magnitude. Finally, the power injected to the system $s_{i, t}=-p_{i, t}-j q_{i, t}$, modelled as a negative constant power load, is calculated as follows

$$
\begin{aligned}
p_{i, t} & =p_{i, t-1}+\tau_{i}^{o}\left(f_{i}^{p}\left(\tilde{v}_{i, t}\right)-p_{i, t-1}\right) \\
q_{i, t} & =q_{i, t-1}+\tau_{i}^{o}\left(f_{i}^{q}\left(\tilde{v}_{i, t}\right)-q_{i, t-1}\right)
\end{aligned}
$$

where $\tau_{i}^{o}$ is a time constant. We conclude the modeling of distribution system elements and control functionality. In the next section, we express the power flow equations in terms of the power injections, by leveraging the incidence matrices of the graph. Expressing the equations in matrix-vector form enhances the study of efficient methods to compute updates, e.g. in the controls or in the loads, as we will see later. 
V. BUILDing $\mathbf{Y}_{\text {Bus AND SOlving The PF EQUATIONS }}$

Let $N:=\sum_{n=1}^{|\mathcal{N}|}\left|\Omega_{n}\right|$ and $L=\sum_{\{n, m\} \in \mathcal{E}}\left|\Omega_{n m}\right|$ denote the total number of nodes and lines in the network, respectively, and by $\mathbf{E}$ we denote the $N \times L$ incidence matrix of the grid. We can map the vector of power flows $\underline{s}$ to the vector of power injections $s_{\text {bus }} \in \mathbb{C}^{N}$ given by the incidence matrix, i.e. $\boldsymbol{s}_{\text {bus }}=\mathbf{E} \underline{\boldsymbol{s}}$. Also, we can express the vectors of nodal state variables as $\underline{\mathbf{u}}=\mathbf{E}^{T} \mathbf{u}, \mathbf{u} \in \mathbb{C}^{N}$ and $\underline{\tilde{\boldsymbol{\theta}}}=\mathbf{E}^{T} \tilde{\boldsymbol{\theta}}, \tilde{\boldsymbol{\theta}} \in \mathbb{C}^{N}$. Multiplying from the left by $\mathbf{E}$ in Eq. (15) and substituting these expressions, we obtain

$$
\boldsymbol{s}_{\text {bus }} \approx \mathbf{E} \mathbf{Y}^{\boldsymbol{u}} \mathbf{E}^{T} \boldsymbol{u}+\mathbf{E} \mathbf{Y}^{\tilde{\theta}} \mathbf{E}^{T} \tilde{\boldsymbol{\theta}}+\mathbf{E} \underline{\boldsymbol{y}}
$$

where the full expression for the bus injections $s_{\text {bus }}$ can be obtained from the ZIP loads. Let us denote by $L_{\mathrm{A}}^{b}$ the total number of phases in the set of $\mathrm{A}$-connected constant- $b$ loads, where $\mathrm{A} \in\{\mathrm{Y}, \Delta\}$ and $b \in\{\mathrm{z}, i, p\}, L_{\mathrm{A}}^{b}:=\sum_{\ell \in \mathcal{L}_{\mathrm{A}}^{b}}\left|\Omega_{\ell}\right|$ and introduce the adjacency matrices $\mathbf{E}_{\mathrm{A}}^{b}$ between the load phases and the set of bus-phases, for the set of $\mathrm{A}$-connected constant- $b$ loads:

$$
\left[\mathbf{E}_{\mathrm{A}}^{b}\right]_{n, \omega}:=\left\{\begin{array}{ll}
1 & \text { if } \omega \in \Omega_{n} \\
0 & \text { else }
\end{array}, \quad \forall n \in \mathcal{N}, \forall \omega \in \Omega_{\ell}, \forall \ell \in \mathcal{L}_{\mathrm{A}}^{b}\right.
$$

We stack in the vectors denoted by $\mathbf{y}_{\mathrm{A}}, \boldsymbol{i}_{\mathrm{A}}$ and $\mathbf{s}_{\mathrm{A}}$ all the vectors of constants needed to define the A-connected ZIP load models.

$$
\begin{aligned}
\mathbf{y}_{\mathrm{A}} & =\left[\begin{array}{c}
\mathbf{y}_{1}^{*} \\
\vdots \\
\mathbf{y}_{\left|\mathcal{L}_{\mathrm{A}}^{*}\right|}^{*}
\end{array}\right], \iota_{\mathrm{A}}=\left[\begin{array}{c}
\iota_{1} \\
\vdots \\
\boldsymbol{\iota}_{\left|\mathcal{L}_{\mathrm{A}}^{i}\right|}
\end{array}\right], \mathbf{s}_{\mathrm{A}}=\left[\begin{array}{c}
\mathbf{s}_{1} \\
\vdots \\
\mathbf{s}_{\left|\mathcal{L}_{\mathrm{A}}^{p}\right|}
\end{array}\right] \\
\mathbf{Y}_{\mathrm{A}} & =\operatorname{diag}\left(\left[\mathbf{Y}_{1}^{*}, \ldots, \mathbf{Y}_{\left|\mathcal{L}_{\mathrm{A}}^{\mathrm{z}}\right|}^{*}\right]\right), \mathbf{I}_{\mathrm{A}}=\operatorname{diag}\left(\left[\mathbf{I}_{1}, \ldots, \mathbf{I}_{\left.\mid \mathcal{L}_{\mathrm{A}}^{i}\right]}\right]\right)
\end{aligned}
$$

and we can express $s_{\text {bus }}$ as follows:

$$
\begin{aligned}
& \boldsymbol{s}_{\text {bus }}:=\mathbf{s}_{\text {load }}+\mathbf{Y}_{\text {load }}^{\boldsymbol{u}} \boldsymbol{u}+\mathbf{Y}_{\text {load }}^{\tilde{\boldsymbol{\theta}}} \tilde{\boldsymbol{\theta}} \\
& \mathbf{s}_{\text {load }}:=\mathbf{E}_{\mathrm{Y}}^{\mathrm{Z}} \mathbf{y}_{\mathrm{Y}}+\mathbf{E}_{\Delta}^{\mathrm{z}} \mathbf{y}_{\Delta}+\mathbf{E}_{\mathrm{Y}}^{i} \iota_{\mathrm{Y}}+\mathbf{E}_{\Delta}^{i} \iota_{\Delta}+\mathbf{E}_{\mathrm{Y}}^{p} \mathbf{s}_{\mathrm{Y}}+\mathbf{E}_{\Delta}^{p} \mathbf{s}_{\Delta} \\
& \mathbf{Y}_{\text {load }}^{\boldsymbol{u}}:=2 \mathbf{E}_{\mathrm{Y}}^{\mathrm{Z}} \mathbf{Y}_{\mathrm{Y}}\left(\mathbf{E}_{\mathrm{Y}}^{\mathrm{Z}}\right)^{T}+\mathbf{E}_{\mathrm{Y}}^{i} \mathbf{I}_{\mathrm{Y}}\left(\mathbf{E}_{\mathrm{Y}}^{i}\right)^{T}+ \\
& \mathbf{E}_{\Delta}^{\mathbf{z}}\left(\mathbf{Y}_{\Delta}+\operatorname{diag}\left(\mathbf{y}_{\Delta}\right)\right)\left(\mathbf{E}_{\Delta}^{\mathbf{z}}\right)^{T}+\mathbf{E}_{\Delta}^{\boldsymbol{i}} \mathbf{I}_{\Delta}\left(\mathbf{E}_{\Delta}^{\boldsymbol{i}}\right)^{T} \\
& \mathbf{Y}_{\text {load }}^{\tilde{\theta}}:=j \mathbf{E}_{\mathrm{Y}}^{i} \mathbf{I}_{\mathrm{Y}}\left(\mathbf{E}_{\mathrm{Y}}^{\boldsymbol{i}}\right)^{T}+j \mathbf{E}_{\Delta}^{\boldsymbol{i}} \mathbf{I}_{\Delta}\left(\mathbf{E}_{\Delta}^{\boldsymbol{i}}\right)^{T} \\
& j \mathbf{E}_{\Delta}^{\mathbf{z}}\left(\mathbf{Y}_{\Delta}-\operatorname{diag}\left(\mathbf{y}_{\Delta}\right)\right)\left(\mathbf{E}_{\Delta}^{\mathbf{z}}\right)^{T}
\end{aligned}
$$

Eq. (37) includes all regulators when $\boldsymbol{\Gamma}_{r}=\mathbb{I}$. For the case when $\boldsymbol{\Gamma}_{r} \neq \mathbb{I}$, we can add the difference $\boldsymbol{\Gamma}_{r}-\mathbb{I}$ to Eq. (37). For that, we define $\mathbf{E}_{r} \in[0,1]^{N \times L_{r}}$ where $L_{r}=\sum_{r \in \mathcal{E}_{r}}\left|\Omega_{r}\right|$, and $\mathbf{Y}_{r}^{u}, \mathbf{Y}_{r}^{\tilde{\theta}}$ are the entries of $\mathbf{Y}^{u}, \mathbf{Y}^{\tilde{\theta}}$ corresponding to the regulators. Including Eq. (40) and the action of the regulator controls in Eq. (37), we split the matrix into real and imaginary parts, and we obtain

$$
\log (\mathbf{V}) \text { 3LPF: } \tilde{\mathbf{s}}_{\text {bus }}=\tilde{\mathbf{Y}}_{\text {bus }} \tilde{\mathbf{x}}_{\text {bus }}
$$

where:

$$
\tilde{\mathbf{s}}_{\text {bus }}=\left[\begin{array}{c}
\Re\left(\mathbf{s}_{\text {load }}-\mathbf{E} \underline{\tilde{y}}\right) \\
\mathfrak{I}\left(\mathbf{s}_{\text {load }}-\mathbf{E} \underline{\tilde{y}}\right)
\end{array}\right], \quad \tilde{\mathbf{x}}_{\text {bus }}=\left[\begin{array}{c}
\boldsymbol{u} \\
\tilde{\boldsymbol{\theta}}
\end{array}\right]
$$

and

$$
\tilde{\mathbf{Y}}_{\text {bus }}=\left(\mathbf{A}+\mathbf{U B U}^{T}\right)
$$

with:

$$
\begin{aligned}
\mathbf{A} & :=\left(\mathbb{I}_{2} \otimes \mathbf{E}\right)\left[\begin{array}{ll}
\mathfrak{R}\left(\mathbf{Y}^{\boldsymbol{u}}\right) & \mathfrak{R}\left(\mathbf{Y}^{\tilde{\boldsymbol{\theta}}}\right) \\
\mathfrak{I}\left(\mathbf{Y}^{\boldsymbol{u}}\right) & \mathfrak{I}\left(\mathbf{Y}^{\tilde{\boldsymbol{\theta}}}\right)
\end{array}\right]\left(\mathbb{I}_{2} \otimes \mathbf{E}\right)^{T} \\
\mathbf{U} & :=\left[\left(\mathbb{I}_{2} \otimes \mathbf{E}_{r}\right),\left(\mathbb{I}_{2} \otimes \mathbf{E}_{\mathrm{Y}}^{\mathrm{Z}}\right),\left(\mathbb{I}_{2} \otimes \mathbf{E}_{\mathbf{Y}}^{\boldsymbol{i}}\right),\left(\mathbb{I}_{2} \otimes \mathbf{E}_{\Delta}^{\mathrm{Z}}\right),\left(\mathbb{I}_{2} \otimes \mathbf{E}_{\Delta}^{\boldsymbol{i}}\right)\right], \\
\mathbf{B} & :=\operatorname{diag}\left(\left[\mathbf{Y}_{\text {reg }}, \mathbf{Y}_{\text {load }}\right]\right), \mathbf{Y}_{\text {reg }}:=\boldsymbol{\Gamma}_{r}^{-} \mathbf{Y}_{r} \boldsymbol{\Gamma}_{r}^{-} \\
\mathbf{Y}_{r} & :=\left[\begin{array}{ll}
\mathfrak{R}\left(\mathbf{Y}_{r}^{\boldsymbol{u}}\right) & \mathfrak{R}\left(\mathbf{Y}_{r}^{\tilde{\boldsymbol{\theta}}}\right) \\
\mathfrak{I}\left(\mathbf{Y}_{r}^{\boldsymbol{u}}\right) & \mathfrak{I}\left(\mathbf{Y}_{r}^{\tilde{\boldsymbol{\theta}}}\right)
\end{array}\right], \boldsymbol{\Gamma}_{r}^{-}:=\left(\mathbb{I}_{2} \otimes \boldsymbol{\Gamma}_{r}\right)-\mathbb{I} \\
\mathbf{Y}_{\text {load }} & :=\operatorname{diag}\left(\left[\mathbf{Y}_{1}, \mathbf{Y}_{2}, \mathbf{Y}_{3}, \mathbf{Y}_{4}\right]\right)
\end{aligned}
$$

and the matrices $\mathbf{Y}_{1}, \mathbf{Y}_{2}, \mathbf{Y}_{3}, \mathbf{Y}_{4}$ are defined as

$$
\begin{aligned}
& \mathbf{Y}_{1}=\left[\begin{array}{ll}
\mathfrak{R}\left(2 \mathbf{Y}_{\mathbf{Y}}\right) & 0 \\
\mathfrak{I}\left(2 \mathbf{Y}_{\mathbf{Y}}\right) & 0
\end{array}\right], \mathbf{Y}_{2}=\left[\begin{array}{ll}
\mathfrak{R}\left(\mathbf{I}_{\mathbf{Y}}\right) & \mathfrak{R}\left(j \mathbf{I}_{\mathbf{Y}}\right) \\
\mathfrak{I}\left(\mathbf{I}_{\mathbf{Y}}\right) & \mathfrak{I}\left(j \mathbf{I}_{\mathbf{Y}}\right)
\end{array}\right], \\
& \mathbf{Y}_{3}=\left[\begin{array}{ll}
\mathfrak{R}\left(\mathbf{Y}_{\Delta}+\operatorname{diag}\left(\mathbf{y}_{\Delta}\right)\right) & \mathfrak{R}\left(j \mathbf{Y}_{\Delta}-j \operatorname{diag}\left(\mathbf{y}_{\Delta}\right)\right) \\
\mathfrak{I}\left(\mathbf{Y}_{\Delta}+\operatorname{diag}\left(\mathbf{y}_{\Delta}\right)\right) & \mathfrak{I}\left(j \mathbf{Y}_{\Delta}-j \operatorname{diag}\left(\mathbf{y}_{\Delta}\right)\right)
\end{array}\right], \\
& \mathbf{Y}_{4}=\left[\begin{array}{ll}
\mathfrak{R}\left(\mathbf{I}_{\Delta}\right) & \mathfrak{R}\left(j \mathbf{I}_{\Delta}\right) \\
\mathfrak{I}\left(\mathbf{I}_{\Delta}\right) & \mathfrak{I}\left(j \mathbf{I}_{\Delta}\right)
\end{array}\right] .
\end{aligned}
$$

The solution to Eq. (41) is calculated as follows

$$
\tilde{\mathbf{x}}_{\text {bus }} \approx \tilde{\mathbf{Y}}_{\text {bus }}^{-1} \tilde{\mathbf{s}}_{\text {bus }}
$$

and we may recover the voltage phasors on each bus $n \in \mathcal{N}$ as follows

$$
\boldsymbol{v}_{n} \approx \boldsymbol{\Delta}_{3}^{(n)} \operatorname{diag}\left(e^{\boldsymbol{u}_{n}}\right) e^{j \tilde{\boldsymbol{\theta}}_{n}}
$$

\section{A. Using Sherman-Morrison-Woodbury for an efficient com- putation of $\mathbf{Y}_{\text {bus }}^{-1}$}

In its traditional form, the Woodbury identity [30] says that the inverse of a rank-k correction of some matrix of the form $\left(\mathbf{A}+\mathbf{U B U}^{T}\right)^{-1}$ can be computed by doing a rank$\mathrm{k}$ correction to the inverse of the original matrix. Initially, this required the matrices $\mathbf{A}, \mathbf{B}, \mathbf{U}$ to be square and $\mathbf{A}, \mathbf{B}$ non-singular. In our case, $\mathbf{A}$ is non-singular for a connected graph. Note that we include all regulators when $\boldsymbol{\Gamma}_{r}=\mathbb{I}$ to avoid disconnecting the graph. In our case, $\mathbf{B}$ may not be invertible, and the incidence matrix $\mathbf{U}$ is non-square. In [31], new expressions were derived that relax these constraints, namely

$$
\begin{aligned}
\left(\mathbf{A}+\mathbf{U B U}^{T}\right)^{-1}= & \mathbf{A}^{-1}- \\
& \mathbf{A}^{-1} \mathbf{U}\left(\mathbb{I}+\mathbf{B} \mathbf{U}^{T} \mathbf{A}^{-1} \mathbf{U}\right)^{-1} \mathbf{B} \mathbf{U}^{T} \mathbf{A}^{-1}
\end{aligned}
$$

Thus, the complexity of calculating the inverse in Eq. (49) lies in calculating the products and the inverse involving $\mathbf{B}$, since it is the only element that is changing over time. The elements that are tied to the topology of the network $\mathbf{A}, \mathbf{U}$ remain unchanged, and can be pre-computed. 


\section{B. $\log (v)$ vs OpenDSS in FLOPS}

It is known that commercial-grade software such as OpenDSS use LU decomposition methods [21] to calculate the inverse of $\mathbf{Y}_{\text {bus }} \in \mathbb{R}^{n \times n}$, where $n$ is also the rank of $\tilde{\mathbf{Y}}_{\text {bus. }}$. In general, the computational complexity of the LU decomposition is $\mathcal{O}\left(\frac{2}{3} n^{3}\right)$, and the inverse takes an additional $\mathcal{O}\left(2 n^{2}\right)$, where $\mathcal{O}\left(n^{2}\right)$ are for the forward substitution and $\mathcal{O}\left(n^{2}\right)$ for the backward substitution. In contrast, using the Woodbury identity to exploit the sparsity of the changes may speed up the process. Let the matrices in Eq. (49) be defined as $\mathbf{A} \in \mathbb{R}^{n \times n}, \mathbf{B} \in \mathbb{R}^{k \times k}, \mathbf{U} \in \mathbb{R}^{n \times k}$, where $k$ is the rank of the correction in Eq. (49). The complexity of calculating the inverse using the Woodbury is partitioned as follows

- The product $\mathbf{B} \mathbf{U}^{T} \mathbf{A}^{-1} \mathbf{U}$ is $\mathcal{O}\left(k^{3}\right)$, since the right-hand side can be precomputed

- The addition $\mathbb{I}+\mathbf{B} \mathbf{U}^{T} \mathbf{A}^{-1} \mathbf{U}$ is $\mathcal{O}(k)$ since $\mathbb{I}$ is the identity matrix with $k$ ones in the diagonal

- The inverse $\left(\mathbb{I}+\mathbf{B U}^{T} \mathbf{A}^{-1} \mathbf{U}\right)^{-1}$ and product $\left(\mathbb{I}+\mathbf{B U}^{T} \mathbf{A}^{-1} \mathbf{U}\right)^{-1} \mathbf{B}$ are $\mathcal{O}\left(k^{3}\right)$ each

- The products $\mathbf{A}^{-1} \mathbf{U}\left(\mathbb{I}+\mathbf{B U}^{T} \mathbf{A}^{-1} \mathbf{U}\right)^{-1}$ and $\left(\mathbb{I}+\mathbf{B U}^{T} \mathbf{A}^{-1} \mathbf{U}\right)^{-1} \mathbf{B} \mathbf{U}^{T} \mathbf{A}^{-1}$ are $\mathcal{O}\left(n k^{2}\right)$ each

- Finally, the subtraction that completes the calculation of the inverse is $\mathcal{O}\left(n^{2}\right)$

And the complexity of each algorithm is given by the following expressions

$$
\begin{aligned}
\text { Log(v) 3LPF: } & \mathcal{O}\left(3 k^{3}+2 n k^{2}+n^{2}+k\right) \\
\text { OpenDSS: } & \mathcal{O}\left(\frac{2}{3} n^{3}+2 n^{2}\right)
\end{aligned}
$$

The performance of the $\log (\mathrm{v}) 3 \mathrm{LPF}$ changes with $k$ which is given as $k=2\left(L_{\mathrm{Y}}^{\mathrm{z}}+L_{\mathrm{Y}}^{i}+L_{\Delta}^{\mathrm{z}}+L_{\Delta}^{i}+2 L_{r}\right)$. To put these results in perspective, we show in Table II the FLOPS needed to compute the inverse of some of the IEEE and Low Voltage (LV) cases, ranging from the IEEE-13 to the IEEE-8500 to show different network sizes. It is shown that the $\log (\mathrm{V}) 3 \mathrm{LPF}$ scales with the size of the network and provides a significant advantage when computing the inverse, with the exception of the IEEE-34 in which there is an abundance of loads and regulators.

TABLE II

\begin{tabular}{|c|c|c|c|c|c|}
\hline Case & Nodes & $\mathbf{k}$ & OpenDSS & $\log (v) 3 L P F$ & Ratio \\
\hline IEEE-13 & 41 & 32 & $3.94 \mathrm{e}+05$ & $2.73 e+05$ & 1.44 \\
\hline IEEE-34 & 95 & 114 & $4.72 \mathrm{e}+06$ & $9.42 \mathrm{e}+06$ & 0.50 \\
\hline IEEE-37 & 117 & 60 & $8.76 \mathrm{e}+06$ & $2.39 \mathrm{e}+06$ & 3.67 \\
\hline IEEE-123 & 278 & 96 & $1.16 \mathrm{e}+08$ & $1.32 \mathrm{e}+07$ & 8.77 \\
\hline American LV & 1170 & 0 & $8.56 \mathrm{e}+09$ & 0 & N/A \\
\hline European LV & 2721 & 0 & $1.08 \mathrm{e}+11$ & 0 & N/A \\
\hline IEEE-8500 & 8531 & 72 & $3.31 \mathrm{e}+12$ & $4.69 \mathrm{e}+08$ & 7060.86 \\
\hline
\end{tabular}
COMPUTATION OF THE MATRIX INVERSE IN FLOPS

It should be noted that the LV test cases do not have capacitor banks, voltage regulators, constant-impedance or constant-current loads. Thus, only constant-power loads are included in these test cases. The $\log (\mathrm{v}) 3 \mathrm{LPF}$ is formulated in terms of power flows and voltages, hence, an update of the system admittance matrix is not necessary since all the changes in boundary conditions are implicitly captured in the vector of power injections.

\section{Simulation CASE STUdy AND RESUlTS}

We present a study to showcase the numerical results that can be obtained by solving the $\log (\mathrm{V}) 3 \mathrm{LPF}$ equations, shown in Eq. (41). We test $\log (\mathrm{V})$ 3LPF on different IEEE test cases, with different sizes, ranging from 41 to 8531 nodes, and different elements and configurations. These tests cases include ZIP loads, substation and split-phase transformers with 120/240 V loads, voltage regulators, capacitor banks, capacitor controls, switches and multi-phase lines.

\section{A. Implementation of the $\log (V) 3 L P F$ solver}

We implement the $\log (\mathrm{v})$ 3LPF equations in Python, and use the SciPy sparse linear algebra library to compute the matrix multiplications. SciPy is built on top of Numpy and uses the $B L A S$ routines to perform linear algebra operations such as vector addition, scalar multiplication, dot products, etc. The $\log (\mathrm{v}) 3 \mathrm{LPF}$ is a wrapper built on top of the python package OpenDSSDirect ${ }^{1}$ to parse the network model. The solver precomputes all the constant matrices in a fixed topology only once. Updates to the loads or the controls are implicitly modeled in the vector of power injections or as updates to the matrix inverse via the Woodbury identity, respectively. It should be noted that under very particular conditions, e.g. in the IEEE-34 test case, a regular Gauss-Jordan elimination may be more efficient than using the Woodbury identity, given $k$ is large enough. During the initialization process the algorithm will calculate the complexity of calculating the inverse in FLOPS, and will chose the optimal method. Also, in the LV test cases, the inverse is only computed once during the initialization, since all loads are constant power and there are no voltage regulators. Thus, all changes are modeled in the vector of power injections, $\tilde{\mathbf{s}}_{\text {bus }}$. The $\log (\mathrm{v}) 3 \mathrm{LPF}$ solver is available through Github $^{2}$.

\section{B. $\log (v) 3 L P F$ vs Lossy DistFlow}

We first compare the Log(V) 3LPF and Lossy DistFlow [16] linearizations with an OpenDSS solution on the IEEE-123 test case. It should be noted that the Lossy DistFlow linearization cannot handle constant-current loads due to the inherent limitations of the DistFlow equations. Also, the DistFlow linearization does not include the modelling on controls or devices other than lines or loads. Therefore, we replace the elements for their line equivalent when possible, and treat constantcurrent loads as constant-power. These simplifications are for comparison purposes, and are not considered in any other analysis in this section. We assume a strong voltage at the feeder-head that is kept constant at 1.02 p.u. We compare the voltage profile on the three phases obtained using the $\log (\mathrm{v})$ 3LPF, Lossy DistFlow and OpenDSS. The results from this comparison are shown in Fig. 4, with RMSE values reported in Table III. The $\log (\mathrm{v})$ 3LPF provides a better estimation

\footnotetext{
${ }^{1}$ https://github.com/dss-extensions/OpenDSSDirect.py

${ }^{2}$ https://github.com/Ignacio-Losada/Log-v-3LPF.git
} 

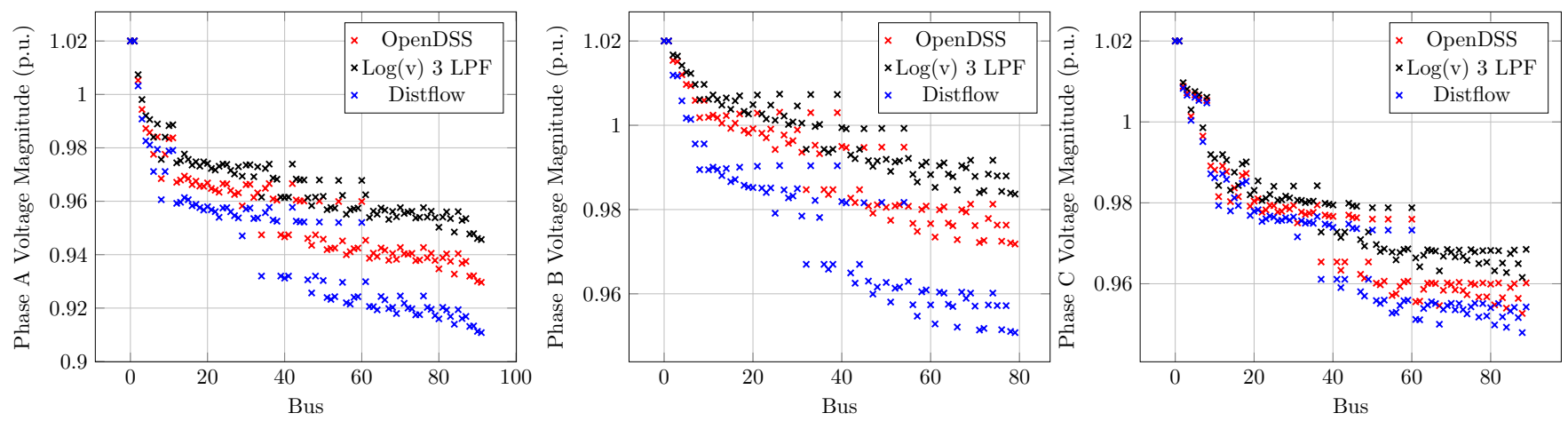

Fig. 4. Comparison between OpenDSS, Log(v) 3LPF and a linearization of the DistFlow equations presented in [16]. The results show the voltage magnitude measured on all phases. Some buses may have less than three phases.The ordering of the buses is based on the hop distance to the feeder head.

TABLE III

RMSE FOR VOLTAGE MAGNitUdeS ACROSS ALL THREE-PHASES FOR IEEE 123 Bus TEST CASE

\begin{tabular}{ccccc}
\hline & Phase A & & Phase B & Phase C \\
\cline { 2 - 2 } Log(v) 3LPF & 0.012 & & 0.008 & 0.004 \\
DistFlow & 0.014 & 0.016 & 0.006 \\
\hline
\end{tabular}

of the voltage on the three phases, with an RMSE less or equal to 0.01 p.u. In general, the $\log (\mathrm{v}) 3 \mathrm{LPF}$ linearization overestimates the voltage magnitude, since it is a linearization around the balanced case. This trend will be seen in the upcoming analysis.

\section{C. $\log (v) 3 L P F$ vs OpenDSS}

In this subsection, we compare the $\log (\mathrm{v}) 3 \mathrm{LPF}$ with OpenDSS on all IEEE test cases from Table II including the modelling of all the elements available. In all cases, we assume a voltage source that is stiff enough, and that can be treated as an infinite source. All wye-delta or delta-wye transformers assume that the secondary terminal and downstream nodes lag the primary by 30 degrees. Switches, and in general voltage regulators, are modelled as lines with a very high admittance. In most cases, distribution lines are three phase and are modeled using their kron reduction. We first present in Table II the results pertaining the accuracy of the $\log (\mathrm{v})$ 3LPF. The results are not included for the North American $\mathrm{LV}$ case due to issues processing the topology and interpreting the geometries. We show the Root Mean Square Error (RMSE) and Mean Absolute Percent Error (MAPE) of the estimates for the voltage magnitude and angle that characterize the phasors.

In general, the errors are less than $3 \%$ of the voltages provided by OpenDSS which we take as the ground truth. We can provide some context for error incurred in the linearization presented here. It has been shown that the earth models used to calculate the impedance of a line, e.g. Carson's equations, may return values for the voltage that differ by more than 0.01 p.u. Therefore, these values are in line with the errors of the $\log (\mathrm{v}) 3 \mathrm{LPF}$.

1) Solving the Power Flow in the IEEE-8500: The IEEE8500 is arguably the most complex system available from the
TABLE IV

ACCURACY OF LOG(V) 3LPF - OPENDSS

\begin{tabular}{ccccc}
\hline \multirow{2}{*}{ Case } & \multicolumn{2}{c}{ Voltage Magnitude } & & Voltage Angle \\
\cline { 2 - 3 } IEEE-13 & RMSE (p.u.) & MAPE (\%) & & RMSE (deg) \\
IEEE-34 & 0.01 & 1.06 & & 1.58 \\
IEEE-37 & 0.03 & 2.46 & \\
IEEE-123 & 0.03 & 2.97 & \\
European LV & 0.02 & 1.64 & \\
IEEE-8500 & 0.00 & 0.38 \\
& 0.02 & 2.45 & & 0.62 \\
& & & & 3.44 \\
\hline
\end{tabular}
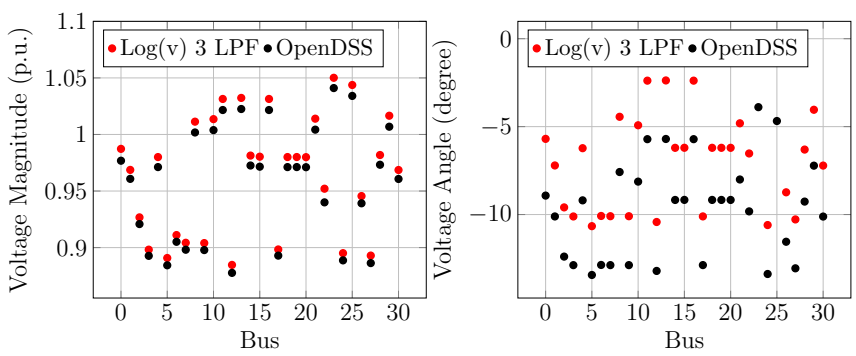

Fig. 5. Comparison between $\log (\mathrm{v}) 3 \mathrm{LPF}$ and OpenDSS, voltage magnitude and angle on phase A for randomly selected nodes of the IEEE 8500 case

IEEE test cases, and perhaps the closest to a real distribution feeder. The IEEE-8500 test case contains multiple triplex lines, that connect the secondary of a center tapped transformer to $120 / 240 \mathrm{~V}$ loads, and are modeled as two-phase lines. In the IEEE- 8500 case, loads are defined as two $120 \mathrm{~V}$ loads connected line-to-neutral. Also, center tapped transformers are defined as single-phase three-winding transformers and its line equivalent can be obtained by applying superposition in Eq. (25). These transformers are connected 7200V line-to-neutral in one of the phases where the connection of the secondary depends on the polarity. To further showcase the $\log (\mathrm{v}) 3 \mathrm{LPF}$ solver, we present in Fig. 5 the voltage levels on phase $A$ that correspond to 30 buses from the 4875 available in the test case. It should be noted that, as previously shown in Fig. 4, the $\log (\mathrm{v}) 3 \mathrm{LPF}$ tends to overestimate the voltage magnitude and angle.

2) Using the $\log (v) 3 L P F$ to control the network: We test the voltage regulator control on the IEEE-13 case. The IEEE- 

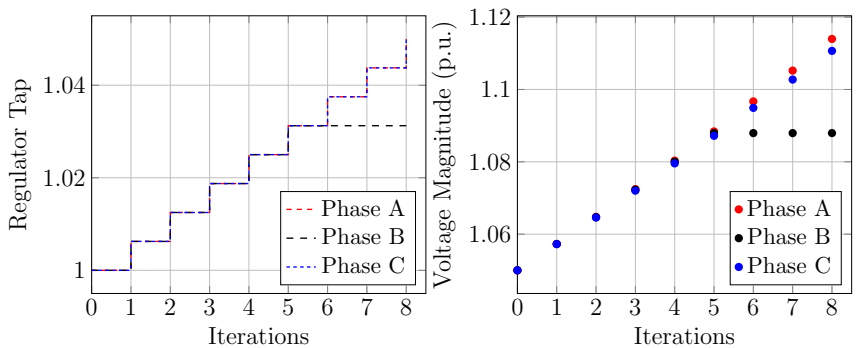

Fig. 6. Voltage regulator controls using the Log(v) 3LPF on the IEEE-13. The figure shows the regulator tap and the downstream bus voltage.

13 contains a single voltage regulator in the network, situated downstream of the substation transformer, keeping a strong voltage at the feeder head. Since there is a single control in the network, the convergence is smooth and controls do not counteract each other. In Fig. 6 we show the actuator applying the controls as well as the resulting impacts of these controls on the downstream bus.

\section{CONCLUSION}

In this paper, we have presented $\log (\mathrm{v}) 3 \mathrm{LPF}$, a linear power flow solver for unbalanced three-phase simulation on distribution systems. The $\log (\mathrm{v}) 3 \mathrm{LPF}$ includes the modeling and control of electrical equipment found on distribution systems. Our results indicate that the $\log (\mathrm{v}) 3 \mathrm{LPF}$ provides an accurate estimate of the voltage phasor, and that the error incurred in on par with other assumptions that are usually made to model distribution lines. Furthermore, we compare our results with commercial-grade software and show that, in general, the $\log (\mathrm{v}) 3 \mathrm{LPF}$ is more efficient that traditional solvers by taking advantage of the sparsity of changes in the boundary conditions. In the context of modeling and control of distribution systems, the $\log (\mathrm{v})$ 3LPF may be used as an efficient solver to learn policies using RL algorithms. This is our goal going forward. Also, we will compare these results with the controls obtained by centralized algorithms, e.g. by solving an OPF problem.

\section{REFERENCES}

[1] J. Ward and H. Hale, "Digital computer solution of power-flow problems [includes discussion]," Transactions of the American Institute of Electrical Engineers. Part III: Power Apparatus and Systems, vol. 75, no. 3, pp. 398-404, 1956.

[2] W. F. Tinney and C. E. Hart, "Power flow solution by newton's method," IEEE Transactions on Power Apparatus and systems, no. 11, pp. 14491460, 1967.

[3] B. Stott and O. Alsac, "Fast decoupled load flow," IEEE transactions on power apparatus and systems, no. 3, pp. 859-869, 1974.

[4] S. Goswami and S. Basu, "Direct solution of distribution systems," in IEE proceedings C (generation, transmission and distribution), vol. 138, no. 1. IET, 1991, pp. 78-88.

[5] C. S. Cheng and D. Shirmohammadi, "A three-phase power flow method for real-time distribution system analysis," IEEE Transactions on Power systems, vol. 10, no. 2, pp. 671-679, 1995.

[6] A. Abur, H. Singh, H. Liu, and W. Klingensmith, "Three phase power flow for distribution systems with dispersed generation," 14th PSCC, Sevilla, vol. 11, no. 3, 2002.

[7] W. H. Kersting, Distribution system modeling and analysis. CRC press, 2017.
[8] J. Carpentier, "Contribution to the economic dispatch problem," Bulletin de la Societe Francoise des Electriciens, vol. 3, no. 8, pp. 431-447, 1962.

[9] S. H. Low, "Convex relaxation of optimal power flow-part i: Formulations and equivalence," IEEE Transactions on Control of Network Systems, vol. 1, no. 1, pp. 15-27, 2014.

[10] T. Smed, G. Andersson, G. Sheble, and L. Grigsby, "A new approach to ac/dc power flow," IEEE Transactions on Power Systems, vol. 6, no. 3, pp. 1238-1244, 1991.

[11] K. Purchala, L. Meeus, D. Van Dommelen, and R. Belmans, "Usefulness of dc power flow for active power flow analysis," in IEEE Power Engineering Society General Meeting, 2005. IEEE, 2005, pp. 454459.

[12] M. E. Baran and F. F. Wu, "Network reconfiguration in distribution systems for loss reduction and load balancing," IEEE Power Engineering Review, vol. 9, no. 4, pp. 101-102, 1989.

[13] B. Sereeter, W. van Westering, C. Vuik, and C. Witteveen, "Linear power flow method improved with numerical analysis techniques applied to a very large network," Energies, vol. 12, no. 21, p. 4078, 2019.

[14] J. Yang, N. Zhang, C. Kang, and Q. Xia, "A state-independent linear power flow model with accurate estimation of voltage magnitude," IEEE Transactions on Power Systems, vol. 32, no. 5, pp. 3607-3617, 2016.

[15] K. Liu, C. Wang, W. Wang, Y. Chen, and H. Wu, "Linear power flow calculation of distribution networks with distributed generation," IEEE Access, vol. 7, pp. 44 686-44 695, 2019.

[16] E. Schweitzer, S. Saha, A. Scaglione, N. G. Johnson, and D. Arnold, "Lossy distflow formulation for single and multiphase radial feeders," IEEE Transactions on Power Systems, vol. 35, no. 3, pp. 1758-1768, 2019.

[17] M. D. Sankur, R. Dobbe, E. Stewart, D. S. Callaway, and D. B. Arnold, "A linearized power flow model for optimization in unbalanced distribution systems," arXiv preprint arXiv:1606.04492, 2016.

[18] A. Bernstein and E. Dall'Anese, "Linear power-flow models in multiphase distribution networks," in 2017 IEEE PES Innovative Smart Grid Technologies Conference Europe (ISGT-Europe). IEEE, 2017, pp. 1-6.

[19] H. Ahmadi, J. R. Mart1, and A. von Meier, "A linear power flow formulation for three-phase distribution systems," IEEE Transactions on Power Systems, vol. 31, no. 6, pp. 5012-5021, 2016.

[20] Z. Li, J. Yu, and Q. Wu, "Approximate linear power flow using logarithmic transform of voltage magnitudes with reactive power and transmission loss consideration," IEEE Transactions on Power Systems, vol. 33, no. 4, pp. 4593-4603, 2017.

[21] E. P. Natarajan, "Klu-a high performance sparse linear solver for circuit simulation problems," Ph.D. dissertation, University of Florida, 2005

[22] D. Montenegro and R. Dugan, "Reference guide, the open distribution system simulator (opendss)," EPRI, March, 2018.

[23] C. Roberts, S.-T. Ngo, A. Milesi, S. Peisert, D. Arnold, S. Saha, A. Scaglione, N. Johnson, A. Kocheturov, and D. Fradkin, "Deep reinforcement learning for der cyber-attack mitigation," in 2020 IEEE International Conference on Communications, Control, and Computing Technologies for Smart Grids (SmartGridComm). IEEE, 2020, pp. 1-7.

[24] S. S. Saha, D. Arnold, A. Scaglione, E. Schweitzer, C. Roberts, S. Peisert, and N. G. Johnson, "Lyapunov stability of smart inverters using linearized distflow approximation," IET Renewable Power Generation, vol. 15 , no. 1 , pp. 114-126, 2021.

[25] R. W. Kenyon, B. Mather, and B.-M. Hodge, "Coupled transmission and distribution simulations to assess distributed generation response to power system faults," Electric Power Systems Research, vol. 189, p. 106746, 2020.

[26] C. Zhao, E. Dall-Anese, and S. H. Low, "Optimal power flow in multiphase radial networks with delta connections," National Renewable Energy Lab.(NREL), Golden, CO (United States), Tech. Rep., 2017.

[27] R. C. Dugan, "Reference guide: The open distribution system simulator (opendss)," Electric Power Research Institute, Inc, vol. 7, p. 29, 2012.

[28] R. Dugan and S. Santoso, "An example of 3-phase transformer modeling for distribution system analysis," in 2003 IEEE PES Transmission and Distribution Conference and Exposition (IEEE Cat. No. 03CH37495), vol. 3. IEEE, 2003, pp. 1028-1032.

[29] I. L. Carreño, R. Ramakrishna, A. Scaglione, D. Arnold, C. Roberts, S.T. Ngo, S. Peisert, and D. Pinney, "Soda: An irradiance-based synthetic solar data generation tool," in 2020 IEEE International Conference on Communications, Control, and Computing Technologies for Smart Grids (SmartGridComm). IEEE, 2020, pp. 1-6.

[30] M. A. Woodbury, Inverting modified matrices. Statistical Research Group, 1950.

[31] H. V. Henderson and S. R. Searle, "On deriving the inverse of a sum of matrices," Siam Review, vol. 23, no. 1, pp. 53-60, 1981. 